\title{
Flavor violating gluino three-body decays at the LHC
}

\author{
A. Bartl, ${ }^{1}$ H. Eberl, ${ }^{2}$ E. Ginina, ${ }^{1}$ B. Herrmann, ${ }^{3}$ K. Hidaka, ${ }^{4}$ W. Majerotto, ${ }^{2}$ and W. Porod ${ }^{5}$ \\ ${ }^{1}$ Universität Wien, Fakultät für Physik, A-1090 Vienna, Austria \\ ${ }^{2}$ Institut für Hochenergiephysik der Österreichischen Akademie der Wissenschaften, A-1050 Vienna, Austria \\ ${ }^{3}$ Deutsches Elektronen-Synchrotron (DESY), Theory Group, D-22603 Hamburg, Germany \\ ${ }^{4}$ Department of Physics, Tokyo Gakugei University, Koganei, Tokyo 184-8501, Japan \\ ${ }^{5}$ Institut für Theoretische Physik und Astrophysik, Universität Würzburg, D-97074 Würzburg, Germany
}

(Received 18 July 2011; published 29 December 2011)

We study the effect of squark generation mixing on gluino production and decays at LHC in the minimal supersymmetric standard model for the case that the gluino is lighter than all squarks and dominantly decays into three particles, $\tilde{g} \rightarrow q \bar{q} \tilde{\chi}_{k}^{0}, q \bar{q}^{\prime} \tilde{\chi}_{l}^{ \pm}$. We assume mixing between the second and the third squark generations in the up-type and down-type squark sectors. We show that this mixing can lead to very large branching ratios of the quark-flavor violating gluino three-body decays despite the strong constraints on quark-flavor violation (QFV) from the experimental data on B mesons. We also show that the QFV gluino decay branching ratios are very sensitive not only to the generation mixing in the squark sector, but also to the parameters of the neutralino and chargino sectors. We show that the branching ratio of the QFV gluino decay $\tilde{g} \rightarrow c \bar{t}(\bar{c} t) \tilde{\chi}_{1}^{0}$ can go up to $\sim 40 \%$. Analogously, that of the QFV decay $\tilde{g} \rightarrow s \bar{b}(\bar{s} b) \tilde{\chi}_{1}^{0}$ can reach $\sim 35 \%$. We find that the rates of the resulting QFV signatures, such as $p p \rightarrow t t \bar{c} \bar{c} E_{\mathrm{T}}^{\mathrm{mis}}$, can be significant at LHC. This could have an important influence on the gluino searches at LHC.

DOI: 10.1103/PhysRevD.84.115026

PACS numbers: $12.60 . \mathrm{Jv}$

\section{INTRODUCTION}

The flavor structure of the quark sector is very well described by the Cabibbo-Kobayashi-Maskawa (CKM) mixing matrix, which is the only source of quark-flavor violation (QFV) in the standard model (SM). In particular, flavor changing neutral current processes, such as $K^{0} \rightarrow$ $\mu^{+} \mu^{-}, B^{0} \rightarrow \mu^{+} \mu^{-}, B \rightarrow X_{s} \gamma, B \rightarrow X_{s} l^{+} l^{-}$etc., are strongly suppressed [1]. They impose strong constraints on the quark generation mixing. Any extension of the SM must therefore respect these constraints.

In supersymmetric (SUSY) extensions of the SM, mixing between different quark flavors in the squark sector, that is not related to the CKM matrix is also possible. Although the mixing between the first and second generation squarks is strongly constrained, there is room for appreciable mixing between the second and third generation of squarks, still obeying the constraints from B meson data. ${ }^{1}$ This is beyond the minimal flavor violation (MFV), where the only source of QFV is the mixing due to the CKM matrix [3-5].

The effects of mixing between the second and third squark generations, especially the mixing between top and charm squarks, have been studied in the minimal supersymmetric standard model (MSSM) for squark production and decays at LHC [6-10]. Squark generation mixing has been investigated in detail in the QFV decays of gluinos, $\tilde{g} \rightarrow \overline{\tilde{u}}_{1,2} c\left(\tilde{u}_{1,2} \bar{c}\right) \rightarrow c \bar{t} \tilde{\chi}_{1}^{0}\left(\bar{c} t \tilde{\chi}_{1}^{0}\right)$, where $\tilde{u}_{1,2}$ are

\footnotetext{
${ }^{1}$ There could also be mixing between the right up-squark and the left top-squark which is hardly constrained [2]. However, we do not consider this mixing here.
}

the lightest squark states and are mixtures of charm and top squarks [11]. There it is assumed that at least one squark is lighter than the gluino, so that the gluino decays first into a real squark (antisquark) and an antiquark (quark). In [11] it is shown that this leads to pronounced edge structures in the charm top-quark invariant mass distribution.

In the present paper we study the QFV gluino decays in the general MSSM assuming that all squarks are heavier than the gluino, so that the gluino dominantly decays into three particles. This will give rise to a very different pattern of QFV gluino decays as compared to the studies in Ref. [11], due to interference effects between the various virtual squark exchange contributions. Moreover, the invariant mass distributions will have different shapes without any edge structure in contrast to the case studied in [11].

We study the mixing between the second and the third generations not only in the up-type squark sector as in Ref. [11], but also in the down-type sector. We investigate QFV gluino decays including those into down-type quark pair plus neutralino, such as $\tilde{g} \rightarrow s \bar{b} \tilde{\chi}_{1}^{0}$. Furthermore, we also investigate in detail the dependence of the QFV gluino decay branching ratios on the neutralino/chargino parameters. We take into account all relevant experimental constraints on the MSSM parameters from B physics and searches for Higgs bosons and SUSY particles, and the theoretical constraints on the trilinear couplings from the vacuum stability conditions.

Recently ATLAS and CMS performed searches for SUSY at LHC with $\sqrt{s}=7 \mathrm{TeV}$ on the basis of total integrated luminosities of $35 \mathrm{pb}^{-1}$ [12,13] and $\sim 1 \mathrm{fb}^{-1}$ $[14,15]$. They found no excess of events over the SM 
expectations and set limits on the squark and gluino masses at $95 \%$ confidence level (C.L.). In the simplified SUSY model with $m_{\tilde{\chi}_{1}^{0}} \approx 0$ (where all SUSY particles other than the gluino and squarks of the first two generations, and $\tilde{\chi}_{1}^{0}$ are decoupled by being given very heavy masses) the limit on the gluino mass is $m_{\tilde{g}} \gtrsim 800 \mathrm{GeV}$ for large $m_{\tilde{q}}$ and that on the squark mass is $m_{\tilde{q}} \gtrsim 850 \mathrm{GeV}$ for large $m_{\tilde{g}}$ [14] (see [15] also). Here, $m_{\tilde{q}}$ is the degenerate mass of the squarks of the first two generations. In the context of the constrained MSSM (or minimal supergravity) the lower limit on the gluino mass is smaller than $\sim 650 \mathrm{GeV}$ for $m_{\tilde{q}} \gtrsim 1.5 \mathrm{TeV}$ and the limit on the squark mass is $m_{\tilde{q}} \gtrsim$ $1.1 \mathrm{TeV}$ for any $m_{\tilde{g}}[15,16]$ (see [14] also). Here again $m_{\tilde{q}}$ is defined to be the degenerate mass of the squarks of the first two generations and the masses of the third generation squarks are (significantly) smaller than this $m_{\tilde{q}}$ in this framework of the constrained MSSM (minimal supergravity). Therefore we will assume a gluino mass of about $1 \mathrm{TeV}$ in our analysis respecting these gluino mass limits.

\section{SQUARK MIXING WITH FLAVOR VIOLATION}

In the MSSM the most general form of the squark mass matrices in the super-CKM basis of $\tilde{q}_{0 \gamma}=$ $\left(\tilde{q}_{1 L}, \tilde{q}_{2 L}, \tilde{q}_{3 L}, \tilde{q}_{1 R}, \tilde{q}_{2 R}, \tilde{q}_{3 R}\right), \quad \gamma=1, \ldots 6, \quad$ where $\left(q_{1}, q_{2}, q_{3}\right)=(u, c, t),(d, s, b)$ is [17]

$$
\mathcal{M}_{\tilde{q}}^{2}=\left(\begin{array}{ll}
\mathcal{M}_{\tilde{q}, L L}^{2} & \mathcal{M}_{\tilde{q}, L R}^{2} \\
\mathcal{M}_{\tilde{q}, R L}^{2} & \mathcal{M}_{\tilde{q}, R R}^{2}
\end{array}\right),
$$

for $\tilde{q}=\tilde{u}, \tilde{d}$, where the $3 \times 3$ matrices read

$$
\begin{aligned}
\mathcal{M}_{\tilde{d}, L L}^{2} & =M_{Q}^{2}+D_{\tilde{d}, L L} \mathbf{1}+\hat{m}_{d}^{2}, \\
\mathcal{M}_{\tilde{u}, L L}^{2} & =V_{\mathrm{CKM}} M_{Q}^{2} V_{\mathrm{CKM}}^{\dagger}+D_{\tilde{u}, L L} \mathbf{1}+\hat{m}_{u}^{2}, \\
\mathcal{M}_{\tilde{d}, R R}^{2} & =M_{D}^{2}+D_{\tilde{d}, R R} \mathbf{1}+\hat{m}_{d}^{2}, \\
\mathcal{M}_{\tilde{u}, R R}^{2} & =M_{U}^{2}+D_{\tilde{u}, R R} \mathbf{1}+\hat{m}_{u}^{2} .
\end{aligned}
$$

Here $M_{Q, U, D}^{2}$ are the Hermitian soft-SUSY-breaking mass matrices of the squarks and $\hat{m}_{u, d}$ are the diagonal mass matrices of up- and down-type quarks. $D_{\tilde{q}, L L}=$ $\cos 2 \beta m_{Z}^{2}\left(T_{3}^{q}-e_{q} \sin ^{2} \theta_{W}\right) \quad$ and $D_{\tilde{q}, R R}=e_{q} \sin ^{2} \theta_{W}$ $\cos 2 \beta m_{Z}^{2}$, where $T_{3}^{q}$ and $e_{q}$ are the isospin and electric charge of the quarks (squarks), respectively, and $\theta_{W}$ is the weak mixing angle. The left-left blocks of the up-type and down-type squark mass matrices are related by the CKM matrix $V_{\mathrm{CKM}}$ due to the $\mathrm{SU}(2)_{L}$ symmetry. Note that $V_{\mathrm{CKM}} M_{Q}^{2} V_{\mathrm{CKM}}^{\dagger} \simeq M_{Q}^{2}$ as $V_{\mathrm{CKM}} \simeq 1$. The off-diagonal blocks of Eq. (1) read

$$
\begin{aligned}
& \mathcal{M}_{\tilde{d}, R L}^{2}=\mathcal{M}_{\tilde{d}, L R}^{2 \dagger}=\frac{v_{1}}{\sqrt{2}} T_{D}^{T}-\mu^{*} \hat{m}_{d} \tan \beta, \\
& \mathcal{M}_{\tilde{u}, R L}^{2}=\mathcal{M}_{\tilde{u}, L R}^{2 \dagger}=\frac{v_{2}}{\sqrt{2}} T_{U}^{T}-\mu^{*} \hat{m}_{u} \cot \beta .
\end{aligned}
$$

Here $T_{U, D}^{T}$ are transposes of $T_{U, D}$ which are the soft-SUSYbreaking trilinear coupling matrices of the up-, down-type squarks: $\mathcal{L}_{\text {int }} \supset-\left(T_{U \alpha \beta} \tilde{u}_{R \beta}^{\dagger} \tilde{u}_{L \alpha} H_{2}^{0}+T_{D \alpha \beta} \tilde{d}_{R \beta}^{\dagger} \tilde{d}_{L \alpha} H_{1}^{0}\right), \mu$ is the Higgsino mass parameter, and $\tan \beta=v_{2} / v_{1}$, where $v_{1,2}=\sqrt{2}\left\langle H_{1,2}^{0}\right\rangle$ are the vacuum expectation values of the neutral Higgs fields. The squark mass matrices are diagonalized by the $6 \times 6$ unitary matrices $R^{\tilde{q}}, \tilde{q}=\tilde{u}$, $\tilde{d}$, such that

$R^{\tilde{q}} \mathcal{M}_{\tilde{q}}^{2}\left(R^{\tilde{q}}\right)^{\dagger}=\operatorname{diag}\left(m_{\tilde{q}_{1}}^{2}, \ldots, m_{\tilde{q}_{6}}^{2}\right) \quad$ with $\quad m_{\tilde{q}_{1}}<\ldots<m_{\tilde{q}_{6}}$.

The physical mass eigenstates $\tilde{q}_{i}, i=1, \ldots, 6$ are given by $\tilde{q}_{i}=R_{i \alpha}^{\tilde{q}} \tilde{q}_{0 \alpha}$.

In accordance with [18] we define the QFV parameters $\delta_{\alpha \beta}^{L L}, \delta_{\alpha \beta}^{u R R}$ and $\delta_{\alpha \beta}^{u R L}(\alpha \neq \beta)$ as follows:

$$
\begin{gathered}
\delta_{\alpha \beta}^{L L} \equiv M_{Q \alpha \beta}^{2} / \sqrt{M_{Q \alpha \alpha}^{2} M_{Q \beta \beta}^{2}}, \\
\delta_{\alpha \beta}^{u R R} \equiv M_{U \alpha \beta}^{2} / \sqrt{M_{U \alpha \alpha}^{2} M_{U \beta \beta}^{2}}, \\
\delta_{\alpha \beta}^{u R L} \equiv\left(v_{2} / \sqrt{2}\right) T_{U \beta \alpha} / \sqrt{M_{U \alpha \alpha}^{2} M_{Q \beta \beta}^{2}} .
\end{gathered}
$$

Here $\alpha, \beta=1,2,3(\alpha \neq \beta)$ denote the quark flavors. The QFV parameters in the up-type squark sector relevant for this study are $\delta_{23}^{L L}, \delta_{23}^{u R R}, \delta_{23}^{u R L}=\left(\delta_{32}^{u L R}\right)^{*}$ and $\delta_{23}^{u L R}=\left(\delta_{32}^{u R L}\right)^{*}$ which are the $\tilde{c}_{L}-\tilde{t}_{L}, \tilde{c}_{R}-\tilde{t}_{R}, \tilde{c}_{R}-\tilde{t}_{L}$ and $\tilde{c}_{L}-\tilde{t}_{R}$ mixing parameters, respectively. For the down-type squark sector we define the QFV parameters as follows $(\alpha \neq \beta)$ :

$$
\begin{gathered}
\delta_{\alpha \beta}^{d R R} \equiv M_{D \alpha \beta}^{2} / \sqrt{M_{D \alpha \alpha}^{2} M_{D \beta \beta}^{2}}, \\
\delta_{\alpha \beta}^{d R L} \equiv\left(v_{1} / \sqrt{2}\right) T_{D \beta \alpha} / \sqrt{M_{D \alpha \alpha}^{2} M_{Q \beta \beta}^{2}} .
\end{gathered}
$$

The QFV parameters in the down-type squark sector relevant for our study are $\delta_{23}^{L L}, \delta_{23}^{d R R}, \delta_{23}^{d R L}=\left(\delta_{32}^{d L R}\right)^{*}$ and $\delta_{23}^{d L R}=\left(\delta_{32}^{d R L}\right)^{*}$ which are the $\tilde{s}_{L}-\tilde{b}_{L}, \tilde{s}_{R}-\tilde{b}_{R}, \tilde{s}_{R}-\tilde{b}_{L}$ and $\tilde{s}_{L}-\tilde{b}_{R}$ mixing parameters, respectively.

In our analysis we neglect mixing between the first two squark generations due to the severe experimental constraints from $\mathrm{K}$ meson physics. We also neglect mixing between the first and third squark generations focusing on the effects of mixing between the second and third generations. We assume all the QFV parameters to be real. These parameters are also subject to the experimental constraints given in Table I.

Furthermore we impose the vacuum stability conditions for the trilinear coupling matrices [28]

$$
\begin{aligned}
& \left|T_{U \alpha \alpha}\right|^{2}<3 Y_{U \alpha}^{2}\left(M_{Q \alpha \alpha}^{2}+M_{U \alpha \alpha}^{2}+m_{2}^{2}\right), \\
& \left|T_{D \alpha \alpha}\right|^{2}<3 Y_{D \alpha}^{2}\left(M_{Q \alpha \alpha}^{2}+M_{D \alpha \alpha}^{2}+m_{1}^{2}\right),
\end{aligned}
$$


TABLE I. Constraints on the MSSM parameters from the $B$-physics experiments relevant mainly for the mixing between the second and the third generations of squarks, and from the Higgs sector. The fourth column shows constraints at $95 \%$ C.L. obtained by combining the experimental error quadratically with the theoretical uncertainty, except for $B\left(B_{s} \rightarrow \mu^{+} \mu^{-}\right)$and $m_{h^{0}}$, which is the mass of the lighter $C P$-even neutral Higgs boson. $R_{B \rightarrow \tau \nu}^{\mathrm{SUSY}} \equiv \frac{B\left(B^{+} \rightarrow \tau^{+} \nu\right)_{\mathrm{SUSY}}}{B\left(B^{+} \rightarrow \tau^{+} \nu\right)_{\mathrm{SM}}} \approx\left[1-\left(\left(m_{B^{+}} \tan \beta\right) / m_{H^{+}}\right)^{2}\right]^{2}$, where $m_{H^{+}}$is the charged Higgs boson mass [19].

\begin{tabular}{lccc}
\hline \hline Observable & Exp. data & Theor. uncertainty & Constr. (95\% C.L.) \\
\hline$\Delta M_{B_{s}}\left[\mathrm{ps}^{-1}\right]$ & $17.77 \pm 0.12(68 \%$ C.L.) [1] & $\pm 3.3(95 \%$ C.L.) [20] & $17.77 \pm 3.31$ \\
$10^{4} \times B(b \rightarrow s \gamma)$ & $3.55 \pm 0.26(68 \%$ C.L.) [21] & \pm 0.23 (68\% C.L.) [22] & $3.55 \pm 0.68$ \\
$10^{6} \times B\left(b \rightarrow s l^{+} l^{-}\right)$ & $1.60 \pm 0.50(68 \%$ C.L.) [23] & \pm 0.11 (68\% C.L.) [24] & $1.60 \pm 1.00$ \\
$(l=e$ or $\mu)$ & $<4.3(95 \%$ C.L.) [25] & & $<4.3$ \\
$10^{8} \times B\left(B_{s} \rightarrow \mu^{+} \mu^{-}\right)$ & $1.68 \pm 0.31(68 \%$ C.L.) [21] & \pm 0.25 (68\% C.L.) [21] & $R_{B \rightarrow \tau \nu}^{\text {SUSY }}=1.40 \pm 0.76$ \\
$10^{4} \times B\left(B^{+} \rightarrow \tau^{+} \nu\right)$ & $>114.4(95 \%$ C.L.) [1,26] & \pm 4.0 [27] & $>110.4$ \\
$m_{h^{0}[\mathrm{GeV}]}$ & & & \\
\hline \hline
\end{tabular}

$$
\begin{aligned}
& \left|T_{U \alpha \beta}\right|^{2}<Y_{U \gamma}^{2}\left(M_{Q \alpha \alpha}^{2}+M_{U \beta \beta}^{2}+m_{2}^{2}\right), \\
& \left|T_{D \alpha \beta}\right|^{2}<Y_{D \gamma}^{2}\left(M_{Q \alpha \alpha}^{2}+M_{D \beta \beta}^{2}+m_{1}^{2}\right),
\end{aligned}
$$

where $\alpha, \beta=1,2,3, \alpha \neq \beta ; \gamma=\operatorname{Max}(\alpha, \beta)$ and $m_{1}^{2}=$ $\left(m_{H^{ \pm}}^{2}+m_{Z}^{2} \sin ^{2} \theta_{W}\right) \sin ^{2} \beta-\frac{1}{2} m_{Z}^{2}, m_{2}^{2}=\left(m_{H^{ \pm}}^{2}+m_{Z}^{2} \sin ^{2} \theta_{W}\right)$ $\cos ^{2} \beta-\frac{1}{2} m_{Z}^{2}$. The Yukawa couplings of the up-type and down-type quarks are $Y_{U \alpha}=\sqrt{2} m_{u_{\alpha}} / v_{2}=\frac{g}{\sqrt{2}} \frac{m_{u_{\alpha}}}{m_{W} \sin \beta}$ $\left(u_{\alpha}=u, c, t\right)$ and $Y_{D \alpha}=\sqrt{2} m_{d_{\alpha}} / v_{1}=\frac{g}{\sqrt{2}} \frac{m_{d_{\alpha}}}{m_{W} \cos \beta}\left(d_{\alpha}=\right.$ $d, s, b)$, with $m_{u_{\alpha}}$ and $m_{d_{\alpha}}$ being the running quark masses at the weak scale and $g$ being the $\mathrm{SU}(2)$ gauge coupling. All soft-SUSY-breaking parameters are also assumed to be given at the weak scale. As SM parameters we take $m_{W}=80.4 \mathrm{GeV}, m_{Z}=91.2 \mathrm{GeV}$ and the on-shell topquark mass $m_{t}=173.3 \mathrm{GeV}$ [29]. We have found that our results shown in the following are fairly insensitive to the precise value of $m_{t}$.

In addition to the constraints in Table I we impose the following limits:

(1) The limit on $\left(m_{A^{0}}, \tan \beta\right)$ from the negative search for neutral MSSM Higgs bosons decaying into a tau pair (i.e. $A^{0} / H^{0} / h^{0} \rightarrow \tau^{+} \tau^{-}$) at LHC [30] with $m_{A^{0}}$ being the mass of the $C P$-odd neutral Higgs boson $A^{0}$.

(2) The experimental limit on SUSY contributions on the electroweak $\rho$ parameter [31]: $\Delta \rho($ SUSY $)<$ 0.0012 .

(3) The LEP limits on the SUSY particle masses [32]: $m_{\tilde{\chi}_{1}^{ \pm}}>103 \mathrm{GeV}, m_{\tilde{\chi}_{1}^{0}}>50 \mathrm{GeV}$, where $m_{\tilde{\chi}_{1}^{ \pm}}$and $m_{\tilde{\chi}_{1}^{0}}$ are the masses of the lighter chargino and the lightest neutralino, respectively.

We take into account all the constraints listed in this section in all plots presented in this article. The constraints on the QFV parameters from $B(b \rightarrow s \gamma), \Delta M_{B_{s}}$ and the vacuum stability conditions are especially important for this study. For the computation of the observables (i.e. physical masses, decay branching ratios, $\Delta M_{B_{s}}$ and $\Delta \rho$ (SUSY)) we use the public code SPHENO v3.0 $[33,34]$.

\section{QFV THREE-BODY DECAYS OF GLUINO}

If all squarks are heavier than the gluino and squark generation mixing occurs only between the second and third generation, one has the following QFV three-particle decays of gluino into quarks and neutralinos $\tilde{\chi}_{i}^{0}, i=1,2$, 3,4 ,

$$
\begin{array}{ll}
\tilde{g} \rightarrow c \bar{t} \tilde{\chi}_{i}^{0}, & \bar{c} t \tilde{\chi}_{i}^{0}, \\
\tilde{g} \rightarrow s \bar{b} \tilde{\chi}_{i}^{0}, & \bar{s} b \tilde{\chi}_{i}^{0} .
\end{array}
$$

We will mainly focus on the decays into $\tilde{\chi}_{1}^{0}$. The corresponding Feynman diagrams for the decay $\tilde{g} \rightarrow c \bar{t} \tilde{\chi}_{i}^{0}$ are shown in Fig. 1. We have interference between the $t$ and the $u$ channel exchanges, as well as between the different $\tilde{u}_{j}$ exchange diagrams. In particular, there can be a strong destructive interference between the $\tilde{u}_{l}$ and $\tilde{u}_{k}$ contributions, if they are mainly $\tilde{c}$ and $\tilde{t}$ mixtures and their masses are similar. For instance, if $\tilde{u}_{l} \sim \cos \theta \tilde{t}_{R}+\sin \theta \tilde{c}_{R}$ and $\tilde{u}_{k} \sim-\sin \theta \tilde{t}_{R}+\cos \theta \tilde{c}_{R}$ then the $\tilde{u}_{l}$ exchange contribution is $\sim \frac{(+\cos \theta \sin \theta)}{\left(p^{2}-m_{\tilde{u}_{u}}^{2}\right)}$ whereas the $\tilde{u}_{k}$ exchange contribution is $\sim \frac{(-\cos \theta \sin \theta)}{\left(p^{2}-m_{\tilde{u}_{k}}^{2}\right)}$. These two contributions almost cancel with each other for $m_{\tilde{u}_{l}} \approx m_{\tilde{u}_{k}}$. The suppression of this cancellation requires a large mass-splitting between the two squarks which can be induced by a large $\tilde{c}_{R}-\tilde{t}_{R}$ mixing term $M_{U_{23}}^{2}$ (or $\delta_{23}^{u R R}$ ) even in case the $\tilde{c}_{R}$ mass parameter $M_{U_{22}}^{2}$ is similar to the $\tilde{t}_{R}$ mass parameter $M_{U_{33}}^{2}$. Moreover, in this case one has a very strong $\tilde{c}_{R}-\tilde{t}_{R}$ mixing. Therefore one can expect sizable QFV decay branching ratios for a large mass-splitting and hence for large values of $\delta_{23}^{u R R}$. For the decays into down-type quarks one has
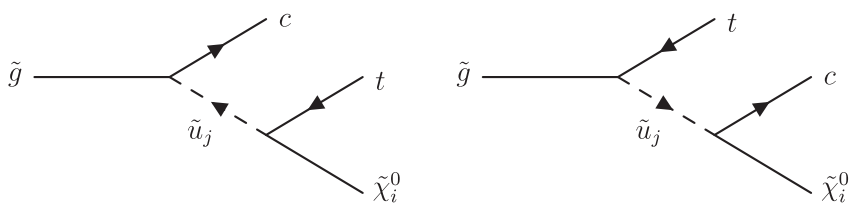

FIG. 1. Feynman diagrams for $\tilde{g} \rightarrow c \bar{t} \tilde{\chi}_{i}^{0}$. 
TABLE II. Weak scale parameters at $Q=1 \mathrm{TeV}$ for our prototype QFV scenario, except for $m_{A^{0}}$ which is the pole mass (i.e. physical mass) of $A^{0}$. All of $T_{U \alpha \alpha}$ and $T_{D \alpha \alpha}$ are 0.

\begin{tabular}{lccccc}
\hline \hline$M_{1}$ & $M_{2}$ & $M_{3}$ & $\mu$ & $\tan \beta$ & $m_{A^{0}}$ \\
\hline $139 \mathrm{GeV}$ & $264 \mathrm{GeV}$ & $800 \mathrm{GeV}$ & $1000 \mathrm{GeV}$ & 10 & $800 \mathrm{GeV}$ \\
\hline \hline \hline & $\alpha=\beta=1$ & $\alpha=\beta=2$ & $\alpha=\beta=3$ \\
\hline \hline$M_{Q \alpha \beta}^{2}$ & $(3150)^{2} \mathrm{GeV}^{2}$ & $(3100)^{2} \mathrm{GeV}^{2}$ & $(3050)^{2} \mathrm{GeV}^{2}$ \\
$M_{U \alpha \beta}^{2}$ & $(3000)^{2} \mathrm{GeV}^{2}$ & $(2200)^{2} \mathrm{GeV}^{2}$ & $(2150)^{2} \mathrm{GeV}^{2}$ \\
$M_{D \alpha \beta}^{2}$ & $(3000)^{2} \mathrm{GeV}^{2}$ & $(2990)^{2} \mathrm{GeV}^{2}$ & $(2980)^{2} \mathrm{GeV}^{2}$ \\
\hline \hline
\end{tabular}

analogous Feynman diagrams with the replacements $c \rightarrow s, t \rightarrow b, \tilde{u}_{j} \rightarrow \tilde{d}_{j}$.

There are also gluino three-body decays into charginos, such as $\tilde{g} \rightarrow c \bar{b}_{1}^{-}, s \bar{t} \tilde{\chi}_{1}^{+}$, etc. We will, however, not discuss them explicitly here, although they are included in our branching ratio calculations.

We calculate the three-particle decay branching ratios of the gluino according to the diagrams in Fig. 1 and their charge conjugated ones, including the QFV couplings given in [6]. As basic SUSY parameters at the weak scale we take $M_{1}, M_{2}, M_{3}, \mu, \tan \beta, m_{A^{0}}, M_{Q \alpha \beta}^{2}, M_{U \alpha \beta}^{2}, M_{D \alpha \beta}^{2}$, $T_{U \alpha \beta}$ and $T_{D \alpha \beta}$, which we assume to be real. Here $M_{1,2,3}$ are the $\mathrm{U}(1), \mathrm{SU}(2)$ and $\mathrm{SU}(3)$ gaugino mass parameters, respectively, and $m_{A^{0}}$ is the pole mass (i.e. physical mass) of the Higgs boson $A^{0}$. We study in detail the QFV scenario based on the parameters of Table II, given at the scale $Q=1 \mathrm{TeV}$, according to the SPA convention [35] except for $m_{A^{0}}$ being the pole mass of $A^{0}$. The scenario of Table II satisfies all the constraints listed in Sec. II; e.g. for the low energy observables we obtain $\Delta M_{B_{s}}=19.01 \mathrm{ps}^{-1}$, $B(b \rightarrow s \gamma)=3.46 \times 10^{-4}, \quad B\left(b \rightarrow s l^{+} l^{-}\right)=1.59 \times 10^{-6}$, $B\left(b \rightarrow s \mu^{+} \mu^{-}\right)=5.02 \times 10^{-9}, \quad R_{B \rightarrow \tau \nu}^{\mathrm{SUSY}}=0.99, m_{h^{0}}=$ $121.1 \mathrm{GeV}, \Delta \rho($ SUSY $)=5.70 \times 10^{-5}$. We add to the parameters of Table II the QFV parameters $\delta_{23}^{L L}, \delta_{23}^{u R R}$, $\delta_{23}^{u R L}, \delta_{23}^{u L R}$ as well as $\delta_{23}^{d R R}, \delta_{23}^{d R L}, \delta_{23}^{d L R}$ (given also at $Q=1 \mathrm{TeV}$ ), and vary them in a range allowed by the constraints listed in Sec. II. The physical masses for the case with all the QFV parameters being zero are shown in Table III. They are calculated from the basic MSSM parameters at the one-loop level, taking into account the complete flavor structure [33]. We have found that these masses are fairly insensitive to the QFV parameters.
Note that in our case QFV left-right mixing effects, i.e. those due to $\delta_{23}^{u R L}, \delta_{23}^{u L R}, \delta_{23}^{d R L}, \delta_{23}^{d L R}$ cannot be significant. We show this for the left-right mixing parameter $\delta_{23}^{u R L}$. Because of the vacuum stability condition (12) we have $\left|T_{U 32}\right|^{2} \lesssim Y_{U 3}^{2}\left(M_{Q 33}^{2}+M_{U 22}^{2}+m_{2}^{2}\right) \approx M_{Q 33}^{2}+M_{U 22}^{2} \approx$ $O\left(10 \mathrm{TeV}^{2}\right)$, because $Y_{U 3} \approx 1$ and $m_{2}^{2} \ll M_{Q 33}^{2}+M_{U 22}^{2}$. Therefore, $\left|\delta_{23}^{u R L}\right|=\frac{v_{2}}{\sqrt{2}} \frac{\left|T_{U 32}\right|}{\sqrt{M_{Q 33}^{2} M_{U 22}^{2}}} \lesssim \frac{v_{2}}{\sqrt{2}} \sqrt{\frac{M_{Q 33}^{2}+M_{U 22}^{2}}{M_{Q 33}^{2} M_{U 22}^{2}}} \approx 0.1$. Analogously, the parameters $\delta_{23}^{u L R}, \delta_{23}^{d R L}, \delta_{23}^{d L R}$ are also constrained to be very small due to the vacuum stability conditions. Therefore, the most relevant QFV parameters in our study are $\delta_{23}^{L L}, \delta_{23}^{u R R}, \delta_{23}^{d R R}$.

Figure 2(a) shows the physical masses of the up-type squarks $\tilde{u}_{1}, \ldots, \tilde{u}_{6}$ as functions of the $\mathrm{QFV}$ parameter $\delta_{23}^{u R R}$, with all the other QFV parameters being zero, for the scenario of Table II. All the constraints mentioned in Sec. II are fulfilled in the shown range. Masses of all the down-type squarks $\tilde{d}_{i}$ are about $3 \mathrm{TeV}$ in this range. For $\left|\delta_{23}^{u R R}\right| \lesssim 0.8$ all squarks are heavier than the gluino. In Fig. 2(b) we show the flavor decomposition of $\tilde{u}_{1}$. For $\left|\delta_{23}^{u R R}\right| \geqslant 0.2, \tilde{u}_{1}$ is practically a full mixture of $\tilde{c}_{R}$ and $\tilde{t}_{R}$. For $\left|\delta_{23}^{u R R}\right| \lesssim 0.88$ the flavor decomposition of $\tilde{u}_{2}$ is similar to that of $\tilde{u}_{1}$ with $\tilde{c}_{R}$ and $\tilde{t}_{R}$ interchanged.

In Fig. 3 we show contours of the branching ratio $B(\tilde{g} \rightarrow$ $\left.c t \tilde{\chi}_{1}^{0}\right) \equiv B\left(\tilde{g} \rightarrow c \bar{t} \tilde{\chi}_{1}^{0}\right)+B\left(\tilde{g} \rightarrow \bar{c} t \tilde{\chi}_{1}^{0}\right)$ in the $\delta_{23}^{L L}-\delta_{23}^{u R R}$ plane together with contours of $10^{4} \times B(b \rightarrow s \gamma)=4.23$ and $\Delta M_{B_{s}}=21.1 \mathrm{ps}^{-1}$, where the other parameters are fixed as in Table II. The branching ratio $B\left(\tilde{g} \rightarrow c t \tilde{\chi}_{1}^{0}\right)$ can reach values larger than $35 \%$. As can be seen, in the range $-0.65 \lesssim \delta_{23}^{L L} \lesssim 0.35$, the $B(b \rightarrow s \gamma)$ and the $\Delta M_{B_{s}}$ constraints are fulfilled. In Ref. [36] it is shown that also the $\rho$-parameter data may constrain the flavor off-diagonal elements of the squark mass matrices, in particular, the $\delta_{\alpha \beta}^{L L}$ entries. However, in our case the $\rho$ parameter practically does not give constraints for two reasons. First, we have negligible left-right squark mixing implying that the left-squark sector is almost decoupled from the rightsquark sector. Second, the mass matrices of the left uptype squarks and the left down-type squarks are related by the $S U(2)_{L}$ symmetry leading to approximately the same masses and mixing matrices for the up-type and down-type squarks.

In Fig. 4 the branching ratios $B\left(\tilde{g} \rightarrow c t \tilde{\chi}_{1}^{0}\right), B\left(\tilde{g} \rightarrow c \bar{c} \tilde{\chi}_{1}^{0}\right)$ and $B\left(\tilde{g} \rightarrow t \bar{t} \tilde{\chi}_{1}^{0}\right)$ are shown as functions of $\delta_{23}^{u R R}$, with the

TABLE III. Physical masses of the particles in the scenario of Table II. $m_{H^{0}}$ is the mass of the heavier $C P$-even neutral Higgs boson $H^{0}$.

\begin{tabular}{lccccr}
\hline \hline$m_{\tilde{\chi}_{1}^{0}}$ & $m_{\tilde{\chi}_{2}^{0}}$ & $m_{\tilde{\chi}_{3}^{0}}$ & $m_{\tilde{\chi}_{4}^{0}}$ & $m_{\tilde{\chi}_{\perp}^{+}}$ & $m_{\tilde{\chi}_{2}^{+}}$ \\
\hline $139 \mathrm{GeV}$ & $281.3 \mathrm{GeV}$ & $1017.9 \mathrm{GeV}$ & $1021.7 \mathrm{GeV}$ & $281.5 \mathrm{GeV}$ & $1022.7 \mathrm{GeV}$ \\
\hline \hline \hline$m_{\tilde{g}}$ & $m_{h^{0}}$ & $m_{H^{0}}$ & $m_{A^{0}}$ & $m_{H^{+}}$ \\
\hline $975 \mathrm{GeV}$ & $121.1 \mathrm{GeV}$ & $800.3 \mathrm{GeV}$ & $800 \mathrm{GeV}$ & $804 \mathrm{GeV}$ \\
\hline \hline
\end{tabular}




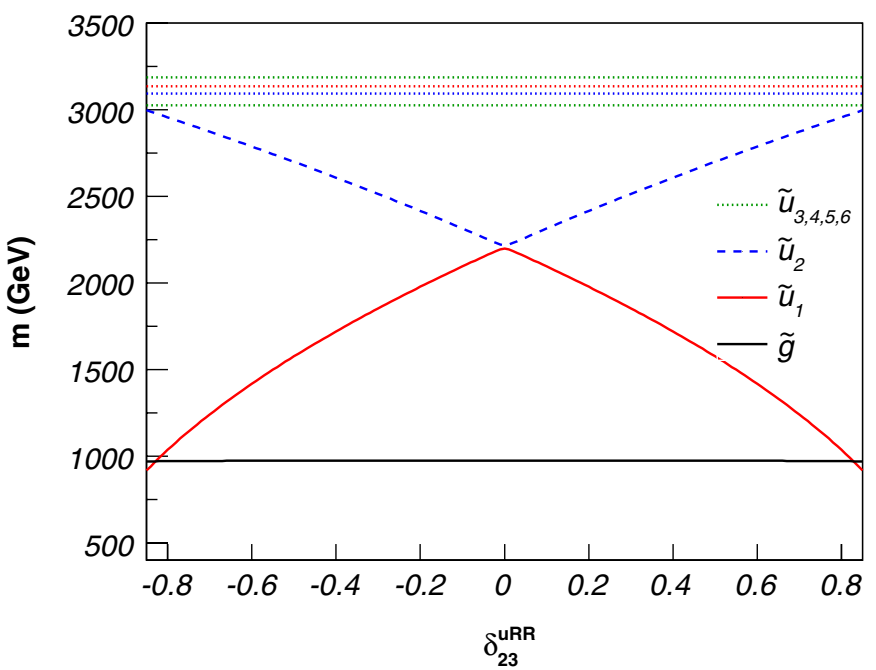

(a)

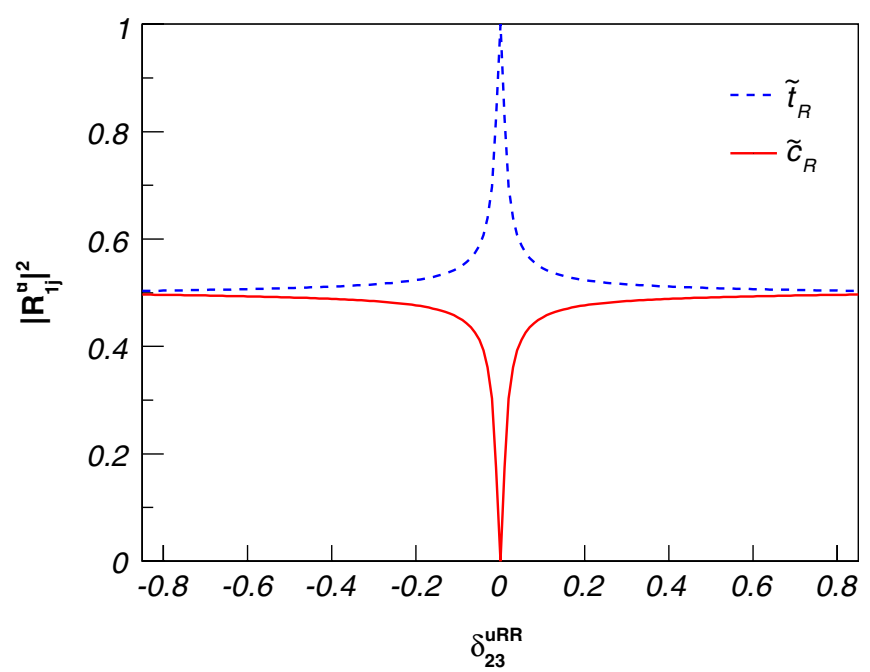

(b)

FIG. 2 (color online). (a) Up-type squark and gluino masses and (b) flavor decomposition of $\tilde{u}_{1}\left[\right.$ i.e. $\left|R_{15}^{\tilde{u}}\right|^{2} \equiv \mid \tilde{c}_{R}$ component $\left.\right|^{2}$ (full red line) and $\left|R_{16}^{\tilde{u}}\right|^{2} \equiv \mid \tilde{t}_{R}$ component $\left.\right|^{2}$ (dashed blue line)] as functions of the QFV parameter $\delta_{23}^{u R R}$, with the other QFV parameters being zero, for the scenario of Table II. In Fig 2(a) the horizontal line around $1000 \mathrm{GeV}$ shows the gluino mass and those around 3000 $\mathrm{GeV}$ show the masses of $\tilde{u}_{3,4,5,6}$.

other QFV parameters being zero and the other parameters fixed as in Table II. All the constraints mention in Sec. II are fulfilled in the shown range. One can see that the QFV decay branching ratio $B\left(\tilde{g} \rightarrow c t \tilde{\chi}_{1}^{0}\right)$ can reach $40 \%$ and that in the range $0.6 \lesssim\left|\delta_{23}^{u R R}\right| \lesssim 0.8$ the QFV decay branching ratio $B\left(\tilde{g} \rightarrow c t \tilde{\chi}_{1}^{0}\right)$ is even larger than the quark-flavor conserving (QFC) branching ratio $B\left(\tilde{g} \rightarrow t \bar{t} \tilde{\chi}_{1}^{0}\right)$. For $\left|\delta_{23}^{u R R}\right| \gtrsim 0.8$, the two-body decays into $\tilde{u}_{1}$ dominate because $\tilde{u}_{1}$ becomes lighter than the gluino [see Fig. 2(a)]. The reason for this large QFV decay branching ratio is as follows: For $0.6 \leqq\left|\delta_{23}^{u R R}\right| \lesssim 0.8$, all squarks other than $\tilde{u}_{1}$ (including down-type squarks) are very heavy [see Fig. 2(a)], which leads to the dominance of the $\tilde{u}_{1}$ exchange contribution in the gluino decays. In this $\delta_{23}^{u R R}$ range the $\tilde{u}_{1}, \tilde{u}_{2}$ are strong mixtures of $\tilde{c}_{R}$ and $\tilde{t}_{R}$ and the mass-splitting between $\tilde{u}_{1}$ and $\tilde{u}_{2}$ is very large, preventing a strong destructive interference between the $\tilde{u}_{1}$ and $\tilde{u}_{2}$ exchange contributions in this $\delta_{23}^{u R R}$ range [see Fig. 2]. This gives the large $\mathrm{QFV}$ decay branching ratio $B\left(\tilde{g} \rightarrow c t \tilde{\chi}_{1}^{0}\right)$. Note that $\tilde{u}_{1}\left(\sim \tilde{c}_{R}+\tilde{t}_{R}\right)$ couples to $\tilde{\chi}_{1}^{0}\left(\approx \tilde{B}^{0}\right)$ and practically does not couple to $\tilde{\chi}_{2}^{0}\left(\approx \tilde{W}^{0}\right)$ and $\tilde{\chi}_{1}^{ \pm}\left(\approx \tilde{W}^{ \pm}\right)$(see Table II). Moreover, $\tilde{\chi}_{3,4}^{0}$ and $\tilde{\chi}_{2}^{ \pm}$are very heavy in the QFV scenario considered here (see Table III.). $\tilde{B}^{0}$ and $\tilde{W}^{0, \pm}$ are the $\mathrm{U}(1)$ and $\mathrm{SU}(2)$ gauginos (the bino and winos), respectively.

On the other hand, as can be seen in Fig. 3, the dependence of $B\left(\tilde{g} \rightarrow c t \tilde{\chi}_{1}^{0}\right)$ on the $\tilde{c}_{L}-\tilde{t}_{L}$ mixing parameter $\delta_{23}^{L L}$ is much weaker than that on $\delta_{23}^{u R R}$. This is mainly due to the fact that in our scenario the left-squarks $\left(\tilde{c}_{L}, \tilde{t}_{L}\right)$ are significantly heavier than the right-squarks $\left(\tilde{c}_{R}, \tilde{t}_{R}\right)$ and that the left-squark coupling to $\tilde{\chi}_{1}^{0}$ ( $\sim$ bino) is small. Hence, the contributions of the left-squark exchanges to $B\left(\tilde{g} \rightarrow c t \tilde{\chi}_{1}^{0}\right)$ are suppressed, leading to the very small effect of the $\tilde{c}_{L}-\tilde{t}_{L}$ mixing parameter $\delta_{23}^{L L}$ on the QFV decay branching ratio. As a consequence, for $\left|\delta_{23}^{u R R}\right| \lesssim 0.4$ the QFV decay branching ratio $B\left(\tilde{g} \rightarrow c t \tilde{\chi}_{1}^{0}\right)$ is smaller than $5 \%$ even for larger allowed values of $\left|\delta_{23}^{L L}\right|$.

The gluino can also have QFV decays into down-type quarks with sizeable branching ratios if the $\delta_{23}^{d R R}$ or $\delta_{23}^{L L}$ are

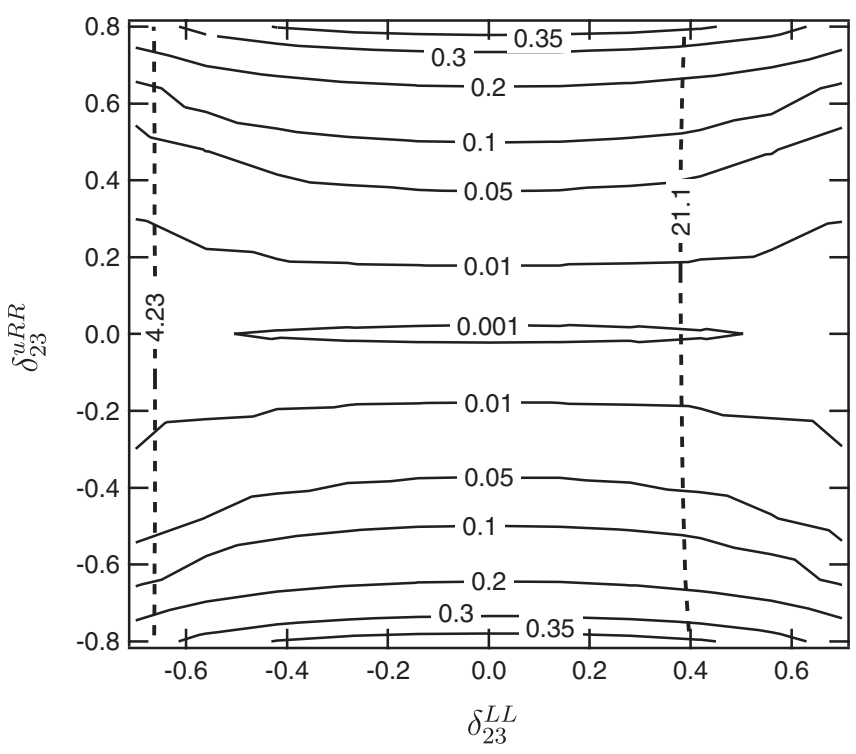

FIG. 3. Contours of the QFV decay branching ratio $B(\tilde{g} \rightarrow$ ct $\left.\tilde{\chi}_{1}^{0}\right)$ in the $\delta_{23}^{L L}-\delta_{23}^{u R R}$ plane with the other QFV parameters being zero, for the scenario of Table II (solid lines). Also shown are the contours of $\Delta M_{B_{s}}=21.1 \mathrm{ps}^{-1}$ and $10^{4} \times B(b \rightarrow s \gamma)=$ 4.23 (dashed lines). The region between the two dashed lines is allowed by all the constraints mentioned in Sec. II, including those from $\Delta M_{B_{s}}$ and $B(b \rightarrow s \gamma)$. 


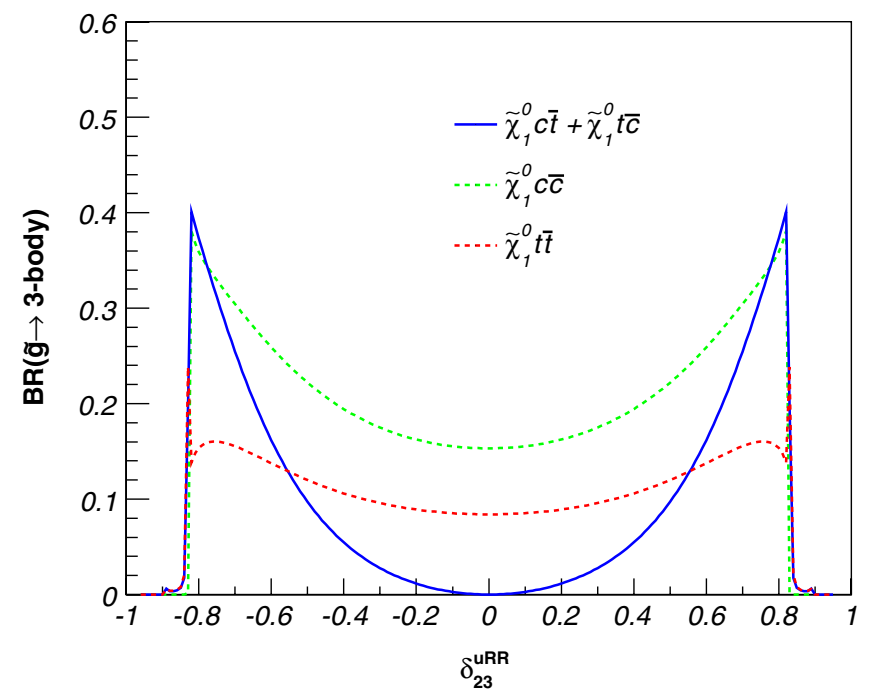

FIG. 4 (color online). The branching ratios of the decays $\tilde{g} \rightarrow$ $c \bar{t} \tilde{\chi}_{1}^{0}+\bar{c} t \tilde{\chi}_{1}^{0}, \quad \tilde{g} \rightarrow c \bar{c} \tilde{\chi}_{1}^{0} \quad$ (upper dashed line) and $\tilde{g} \rightarrow t \bar{t} \tilde{\chi}_{1}^{0}$ (lower dashed line) as functions of $\delta_{23}^{u R R}$ for the other QFV parameters being zero and the other parameters are fixed as in Table II.

unequal to zero. As an example, in Fig. 5 we show a contour plot of the QFV decay branching ratio $B(\tilde{g} \rightarrow$ $\left.s b \tilde{\chi}_{1}^{0}\right) \equiv B\left(\tilde{g} \rightarrow s \bar{b} \tilde{\chi}_{1}^{0}\right)+B\left(\tilde{g} \rightarrow \bar{s} b \tilde{\chi}_{1}^{0}\right)$ in the $\delta_{23}^{d R R}-\delta_{23}^{L L}$ plane for the scenario of Table II. The dashed contour lines for $\Delta M_{B_{s}}=14.5 \mathrm{ps}^{-1}$ and $21.1 \mathrm{ps}^{-1}$ show the region $-0.38 \lesssim \delta_{23}^{L L} \lesssim 0.12$ allowed by all the constraints

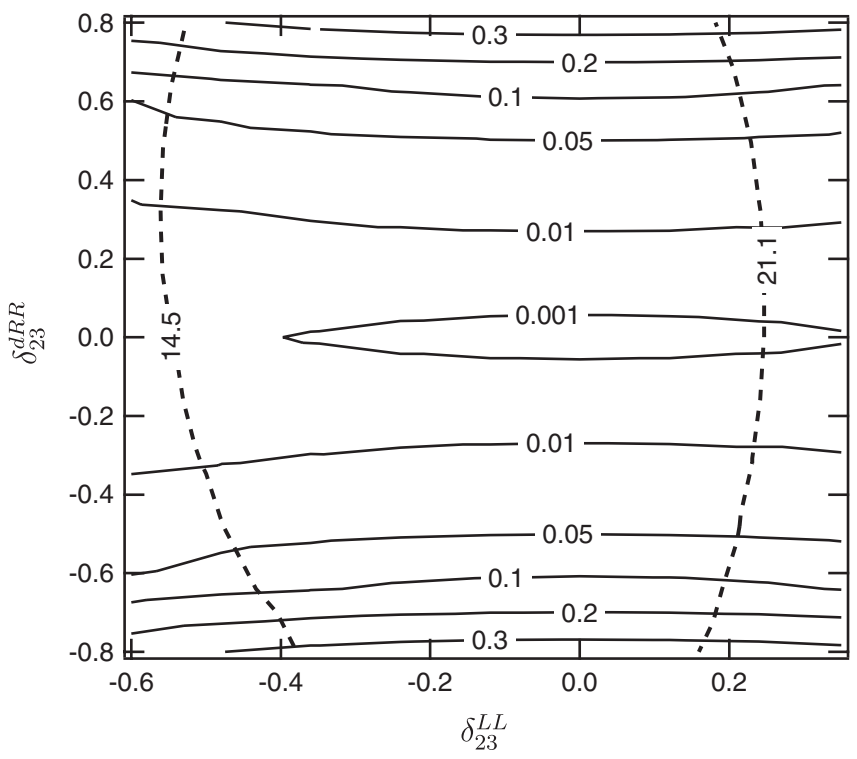

FIG. 5. Contours of the QFV decay branching ratio $B(\tilde{g} \rightarrow$ $\left.s b \tilde{\chi}_{1}^{0}\right)$ in the $\delta_{23}^{L L}-\delta_{23}^{d R R}$ plane, with the other QFV parameters being zero for the scenario of Table II, but with the values of $M_{U \alpha \alpha}^{2}$ and $M_{D \alpha \alpha}^{2}$ interchanged (solid lines). Also shown are the contour lines for $\Delta M_{B_{s}}=14.5 \mathrm{ps}^{-1}$ and $\Delta M_{B_{s}}=21.1 \mathrm{ps}^{-1}$ (dashed lines). The region between the two dashed lines is allowed by all the constraints mentioned in Sec. II, including the $\Delta M_{B_{s}}$ constraint. mentioned in Sec. II, including the $B(b \rightarrow s \gamma)$ constraint. Note that in this case the $\Delta M_{B_{s}}$ constraint is significantly stronger than the $B(b \rightarrow s \gamma)$ constraint in the whole $\delta_{23}^{L L}-\delta_{23}^{d R R}$ plane. $B\left(\tilde{g} \rightarrow s b \tilde{\chi}_{1}^{0}\right)$ can reach values larger than $30 \%$. The reason for this sizable QFV decay branching ratio is similar to that for the large QFV decay branching ratio $B\left(\tilde{g} \rightarrow c t \tilde{\chi}_{1}^{0}\right)$. The dependence of this $\mathrm{QFV}$ decay branching ratio on $\delta_{23}^{L L}$ is again much weaker than that on $\delta_{23}^{d R R}$

We also would like to note that we have found a scenario giving QFV three-body decay branching ratios $B\left(\tilde{g} \rightarrow c t \tilde{\chi}_{1}^{0}\right)\left(\right.$ or $\left.B\left(\tilde{g} \rightarrow s b \tilde{\chi}_{1}^{0}\right)\right)$ of about $50 \%$ for a gluino mass of $\sim 1 \mathrm{TeV}$, still satisfying all the relevant constraints. In such a scenario, however, the heavier squarks have masses of about $6 \mathrm{TeV}$, while the lightest squark heavier than the gluino has a mass of about $1 \mathrm{TeV}$.

\section{INFLUENCE OF THE NEUTRALINO/ CHARGINO PARAMETERS ON THE QFV THREE-BODY GLUINO DECAYS}

As the squark generation mixing enters into the squarkquark-neutralino/chargino couplings, here we study how the pattern of the QFV gluino decays depends on the parameters of the neutralino-chargino sector. First we show in Fig. 6 a contour plot of the branching ratio $B\left(\tilde{g} \rightarrow c t \tilde{\chi}_{1}^{0}\right)$ in the $\mu-M_{2}$ plane for $\delta_{23}^{u R R}=0.8$, the other QFV parameters being zero, and the other parameters fixed as in Table II. In the whole plane $m_{\tilde{g}} \approx 972 \mathrm{GeV}$.

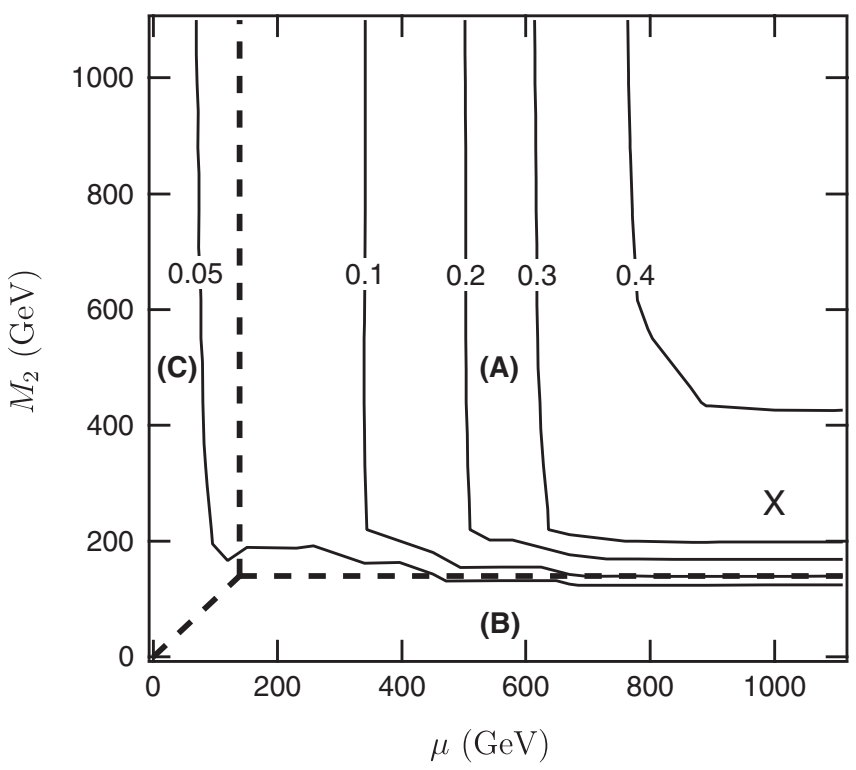

FIG. 6. Contour plot for $B\left(\tilde{g} \rightarrow c t \tilde{\chi}_{1}^{0}\right)$ (solid lines) in the $\mu-$ $M_{2}$ plane for $\delta_{23}^{u R R}=0.8$, the other QFV parameters being zero, and the other parameters specified as in Table II with $M_{1}=$ $139 \mathrm{GeV}$. Region (A): binolike LSP region; region (B): winolike LSP region; region (C): Higgsino-like LSP region. The point " $\mathrm{X}$ " corresponds to our reference scenario given in Table II: $M_{2}=264 \mathrm{GeV}, \mu=1000 \mathrm{GeV}$. 
As one can see, this branching ratio is larger than $10 \%$ for $\mu \gtrsim 350 \mathrm{GeV}$. We indicate the regions where the lightest supersymmetric particle (LSP) is bino-, wino-, or Higgsino-like. The largest QFV decay branching ratio $B\left(\tilde{g} \rightarrow c t \tilde{\chi}_{1}^{0}\right)$ is in the binolike LSP region reaching up to $\sim 40 \%$.

In the following we discuss the dependence of the QFV decay branching ratios of the gluino on the Higgsino mass parameter $\mu$ in more detail. In Fig. 7 we show this dependence for $\delta_{23}^{u R R}=0.8$, the other QFV parameters being zero, and the other parameters fixed as in Table II. In the whole range $m_{\tilde{s}} \approx 972 \mathrm{GeV}$ and the constraints mentioned in Sec. II are satisfied. The branching ratios of the QFV gluino decays $\tilde{g} \rightarrow c \bar{t} \tilde{\chi}_{i}^{0}+\bar{c} t \tilde{\chi}_{i}^{0}, i=1, \ldots, 4$ are shown in Fig. 7(a). For $\mu \gtrsim M_{1}$ the bino component of $\tilde{\chi}_{1}^{0}$ is increasing and hence the branching ratio of $\tilde{g} \rightarrow$ $c \bar{t}(\bar{c} t) \tilde{\chi}_{1}^{0}$ increases. For $\mu \gtrsim 700 \mathrm{GeV}$ this branching ratio is about $36 \%$, being roughly a factor of 2 larger than that for $\tilde{g} \rightarrow t \bar{t} \tilde{\chi}_{1}^{0}$. For $|\mu| \lesssim M_{1}, \tilde{\chi}_{1}^{0}$ and $\tilde{\chi}_{2}^{0}$ are essentially Higgsinos, and the branching ratio of $\tilde{g} \rightarrow c \bar{t}(\bar{c} t) \tilde{\chi}_{1}^{0}$ is less than $10 \%$. The reason is that the QFV decays into the higher neutralinos and the charginos become more important. Note that $|\mu| \lesssim 100 \mathrm{GeV}$ is excluded by the LEP limit on the $m_{\tilde{\chi}_{1}^{ \pm}}$. Furthermore, the final state $c \bar{t} E_{\mathrm{T}}^{\mathrm{mis}}+\bar{c} t E_{\mathrm{T}}^{\mathrm{mis}}$ can contain contributions from the higher neutralino modes $c \bar{t}(\bar{c} t) \tilde{\chi}_{i}^{0}, i \geq 2$, with the invisible decays of $\tilde{\chi}_{i}^{0} \rightarrow \tilde{\chi}_{1}^{0} \nu \bar{\nu}$, where $E_{\mathrm{T}}^{\mathrm{mis}}$ is missing transverse energy. Therefore, we show in Fig. 7(b) a plot where these contributions are included (dashed red line). One can clearly see that for $\mu \lesssim 320 \mathrm{GeV}$ the contributions of the invisible decays of the higher neutralinos [see Fig. 7(a)] are important. For comparison in Fig. 7(b) the QFC branching ratios $B\left(\tilde{g} \rightarrow c \bar{c} \tilde{\chi}_{1}^{0}\right)$ and $B\left(\tilde{g} \rightarrow t \bar{t} \tilde{\chi}_{1}^{0}\right)$ are also shown.

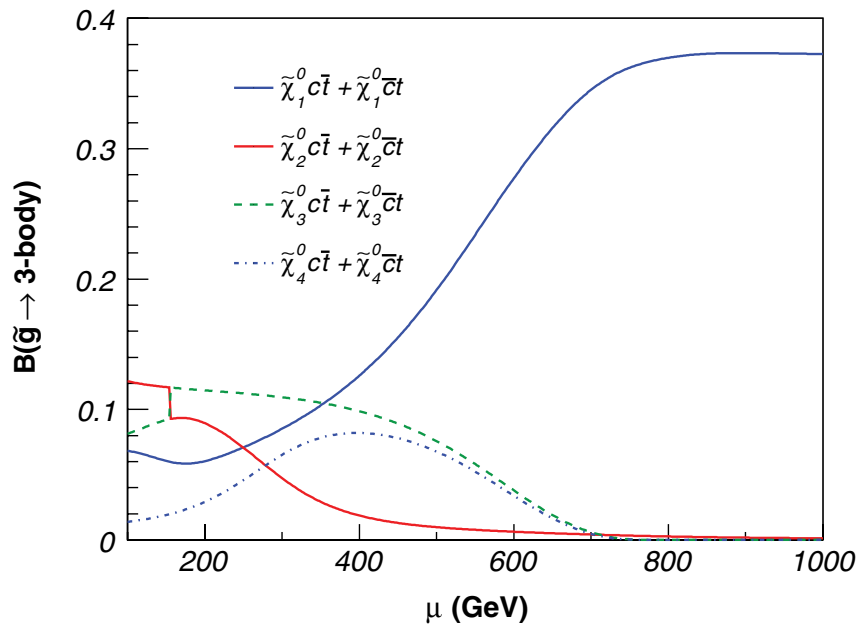

(a)
The situation is quite different if $M_{1}>M_{2}$. In this case, for $\mu \gtrsim M_{2}$ the $\tilde{\chi}_{1}^{0}$ is essentially a wino which does not couple to $\tilde{c}_{R}$ and $\tilde{t}_{R}$ leading to a small branching ratio of $\tilde{g} \rightarrow c \bar{t}(\bar{c} t) \tilde{\chi}_{1}^{0}$. On the other hand, a large branching ratio of $\tilde{g} \rightarrow c \bar{t}(\bar{c} t) \tilde{\chi}_{2}^{0}$ can be expected, because for $|\mu| \geqslant M_{1}, \tilde{\chi}_{2}^{0}$ becomes binolike.

Next we discuss the dependence of the QFV gluino decay branching ratios on the $\mathrm{SU}(2)$ gaugino mass parameter $M_{2}$. In Fig. 8 we show this dependence fixing $M_{1}=$ $264 \mathrm{GeV}, \mu=600 \mathrm{GeV}, \delta_{23}^{u R R}=0.8$, the other QFV parameters being zero, and the other parameters fixed as in Table II. In the shown range $m_{\tilde{g}} \approx 972 \mathrm{GeV}$ and all the constraints mentioned in Sec. II are satisfied. As just explained above, in Fig. 8(a), for $M_{2} \lesssim M_{1}$ the branching ratio of the decay $\tilde{g} \rightarrow c \bar{t}(\bar{c} t) \tilde{\chi}_{1}^{0}$ is almost zero, while that for $\tilde{g} \rightarrow c \bar{t}(\bar{c} t) \tilde{\chi}_{2}^{0}$ is large $(\approx 24 \%)$. For $M_{2} \gtrsim M_{1}$ the roles of $\tilde{\chi}_{1}^{0}$ and $\tilde{\chi}_{2}^{0}$ are interchanged and therefore the decay $\tilde{g} \rightarrow c \bar{t}(\bar{c} t) \tilde{\chi}_{1}^{0}$ becomes dominant with a branching ratio of about $25 \%$. Note that the range $M_{2} \lesssim 100 \mathrm{GeV}$ is excluded by the LEP chargino mass limit mentioned in Sec. II: $m_{\tilde{\chi}_{1}^{ \pm}}>103 \mathrm{GeV}$. At $M_{2} \approx \mu$ there is again a level crossing. The $\tilde{\chi}_{4}^{0}$ is Higgsino-like for $M_{2} \lesssim \mu$ and becomes winolike for $M_{2} \gtrsim \mu$.

In Fig. 8(b) the branching ratios for the decays $\tilde{g} \rightarrow c \bar{b}(\bar{c} b) \tilde{\chi}_{k}^{ \pm}$as a function of $M_{2}$ are shown. The level crossing of $\tilde{\chi}_{1}^{ \pm}$and $\tilde{\chi}_{2}^{ \pm}$at $M_{2} \approx \mu$ is clearly seen.

In the following we discuss in more detail a typical scenario with a Higgsino-like LSP $\left(\mu<M_{1}, M_{2}\right)$ and one with a winolike $\operatorname{LSP}\left(M_{2}<M_{1}, \mu\right)$. As an example for the Higgsino-like LSP scenario, we choose the parameters as given in Table IV(a), with the squark mass parameters as in Table II. We fix the QFV parameter $\delta_{23}^{u R R}=0.8$ and the other QFV parameters equal zero. In this scenario

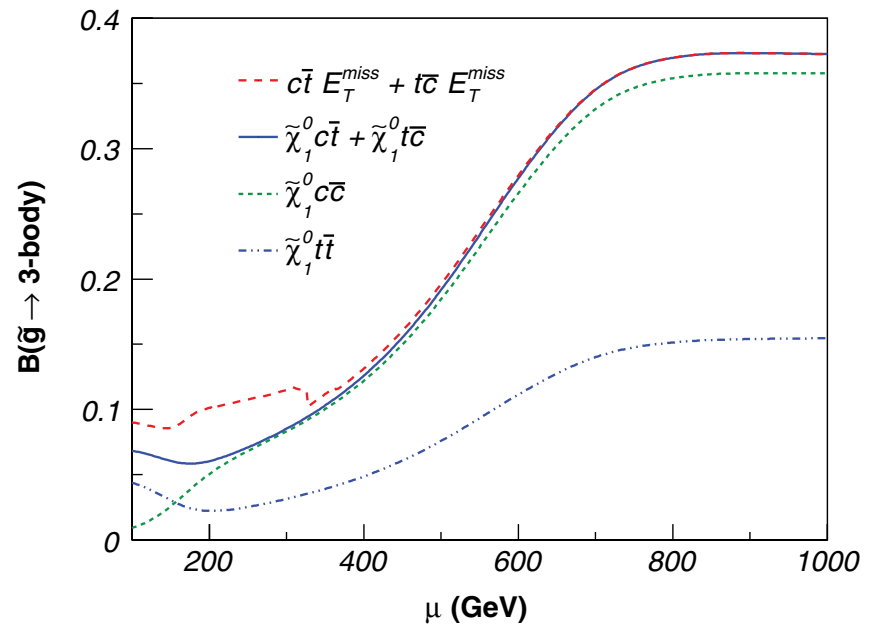

(b)

FIG. 7 (color online). The $\mu$ dependence of the QFV and QFC gluino decay branching ratios for $\delta_{23}^{u R R}=0.8$, the other QFV parameters being zero, for the scenario given in Table II. (a) Branching ratios of the decays $\tilde{g} \rightarrow c \bar{t} \tilde{\chi}_{i}^{0}+\bar{c} t \tilde{\chi}_{i}^{0}, i=1, \ldots, 4$ as a function of $\mu$. Note that $B\left(\tilde{g}->c \bar{t} \tilde{\chi}_{1}^{0}+\bar{c} t \tilde{\chi}_{1}^{0}\right)>B\left(\tilde{g}->c \bar{c} c \tilde{h} i_{2}^{0}+\bar{c} t \tilde{\chi}_{2}^{0}\right)$ for $\mu \geqslant 250$. (b) Branching ratios of the decays $\tilde{g} \rightarrow c \bar{c} \tilde{\chi}_{1}^{0}$, $\tilde{g} \rightarrow t \bar{t} \tilde{\chi}_{1}^{0}, \tilde{g} \rightarrow c \bar{t} \tilde{\chi}_{1}^{0}+\bar{c} t \tilde{\chi}_{1}^{0}$ and $\tilde{g} \rightarrow c \bar{t} E_{\mathrm{T}}^{\mathrm{mis}}+\bar{c} t E_{\mathrm{T}}^{\mathrm{mis}}$ as a function of $\mu$. 


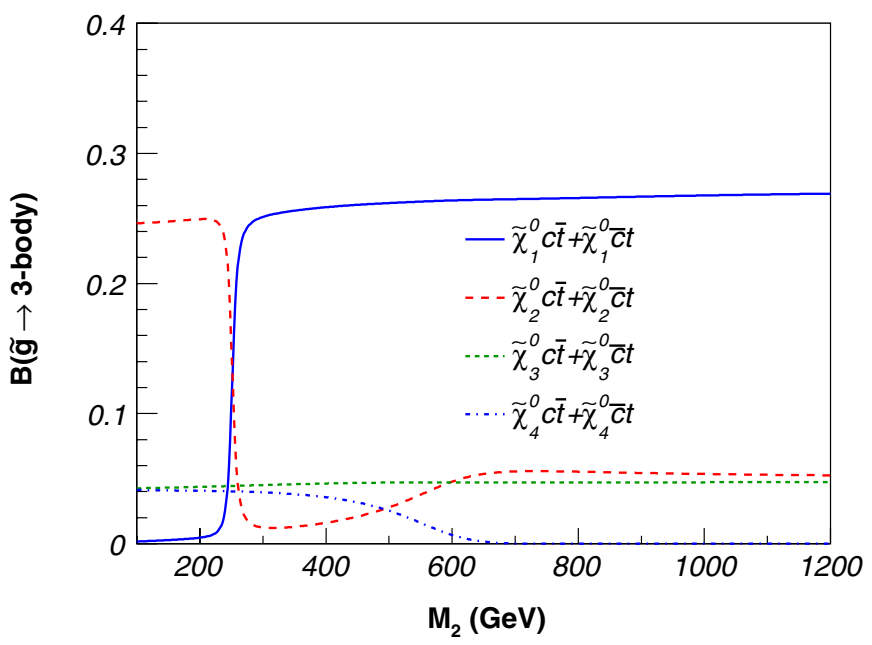

(a)

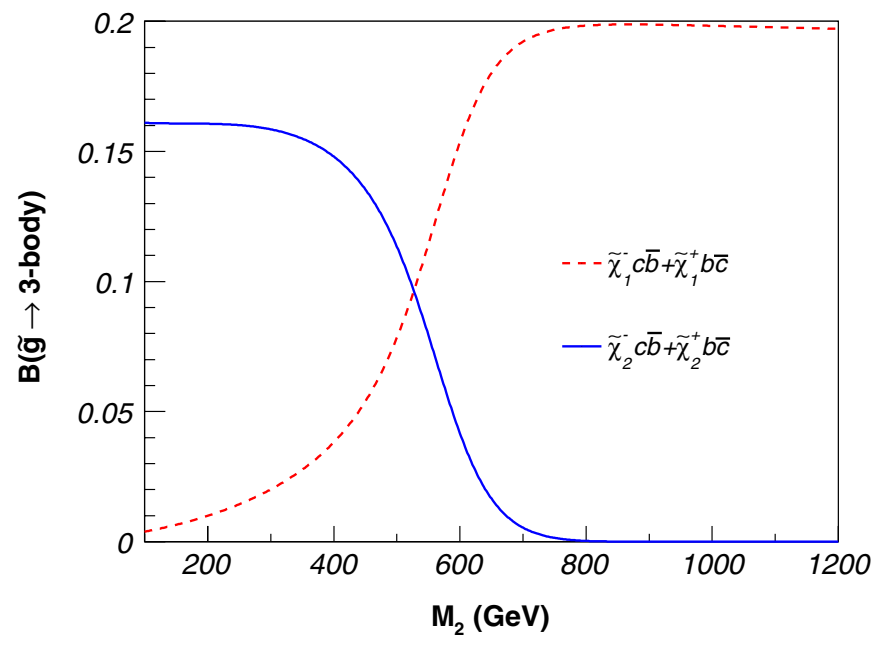

(b)

FIG. 8 (color online). The $M_{2}$ dependence of the QFV gluino decay branching ratios for $M_{1}=264 \mathrm{GeV}, \mu=600 \mathrm{GeV}, \delta_{23}^{u R R}=$ 0.8 , the other QFV parameters being zero, and the other parameters fixed as in Table II. (a) Branching ratios of the decays $\tilde{g} \rightarrow$ $c \bar{t} \tilde{\chi}_{i}^{0}+\bar{c} t \tilde{\chi}_{i}^{0}, i=1, \ldots, 4$ as a function of $M_{2}$. (b) Branching ratios of the decays $\tilde{g} \rightarrow c \bar{b} \tilde{\chi}_{k}^{-}+\bar{c} b \tilde{\chi}_{k}^{+}, k=1,2$ as a function of $M_{2}$.

all experimental and theoretical constraints mentioned in Sec. II are fulfilled. The relevant masses for the neutralinos and the charginos are given in Table IV(b). We show the most important QFV decay branching ratios in Table IV(c). In this scenario $\tilde{\chi}_{1,2}^{0}$ and $\tilde{\chi}_{1}^{ \pm}$are almost Higgsinos and hence their couplings to $\tilde{u}_{1}\left(\sim \tilde{c}_{R}+\tilde{t}_{R}\right)$ are significantly enhanced by the large top-quark Yukawa coupling, which results in the sizable branching ratios of the QFV decays into $\tilde{\chi}_{1,2}^{0}$ and $\tilde{\chi}_{1}^{ \pm}$. Since $B\left(\tilde{\chi}_{2}^{0} \rightarrow \tilde{\chi}_{1}^{0} \nu \bar{\nu}\right)$ is relatively large $(=18.4 \%)$, the sum of the branching ratios for the $\tilde{g}$ decays into the final states $\left(c \bar{t}+E_{\mathrm{T}}^{\mathrm{mis}}\right)$ and $\left(\bar{c} t+E_{\mathrm{T}}^{\mathrm{mis}}\right)$ is sizable $(=9.0 \%)$. The leptonic $\tilde{\chi}_{1}^{ \pm}$decays $\tilde{\chi}_{1}^{ \pm} \rightarrow \mu^{ \pm} \nu_{\mu} \tilde{\chi}_{1}^{0}$ and $\tilde{\chi}_{1}^{ \pm} \rightarrow e^{ \pm} \nu_{e} \tilde{\chi}_{1}^{0}$ have a branching ratio of approximately $11.2 \%$ each, because $W$ exchange dominates. Therefore, from the gluino decays into $\tilde{\chi}_{1}^{ \pm}$one gets final states $b \bar{c}(\bar{b} c)+\mu^{ \pm}\left(e^{ \pm}\right)+E_{\mathrm{T}}^{\mathrm{mis}}$ with a branching ratio of $5.0 \%$. This has to be compared with the expectation from MFV which is of the order of $10^{-4}$, as it is proportional to $\left|V_{c b}\right|^{2}$.

Next we discuss a scenario where the LSP is winolike, with the parameters as given in Table $\mathrm{V}(\mathrm{a})$, the squark mass parameters as in Table II, $\delta_{23}^{u R R}=0.8$ and the other QFV parameters being zero. Again, all the constraints are satisfied. The masses of the neutralinos and charginos are given in Table $\mathrm{V}(\mathrm{b})$. The relevant QFV gluino decay branching ratios are shown in Table $V(c)$. As in this case $B\left(\tilde{\chi}_{2}^{0} \rightarrow \tilde{\chi}_{1}^{0} \nu \bar{\nu}\right)=4.1 \%$, the sum of the branching ratios for the final states $\left(c \bar{t}+E_{\mathrm{T}}^{\mathrm{mis}}\right)$ and $\left(\bar{c} t+E_{\mathrm{T}}^{\mathrm{mis}}\right)$ is $6 \%$. As $B\left(\tilde{\chi}_{1}^{+} \rightarrow \mu^{+} \nu_{\mu} \tilde{\chi}_{1}^{0}\right)=B\left(\tilde{\chi}_{1}^{+} \rightarrow e^{+} \nu_{e} \tilde{\chi}_{1}^{0}\right)=13.2 \%$, one has $B\left(\tilde{g} \rightarrow b \bar{c} \mu^{+} \nu_{\mu} \tilde{\chi}_{1}^{0}\right)=B\left(\tilde{g} \rightarrow \bar{b} c \mu^{-} \bar{\nu}_{\mu} \tilde{\chi}_{1}^{0}\right)=B(\tilde{g} \rightarrow$

TABLE IV. Weak scale parameters at $Q=1 \mathrm{TeV}$ (except for $m_{A^{0}}$ being the pole mass), the corresponding neutralino and chargino masses and some important branching ratios for a scenario with a Higgsino-like LSP, where $m_{\tilde{g}}=972 \mathrm{GeV}$.

(a) Weak scale parameters at $Q=1 \mathrm{TeV}$.

\begin{tabular}{lccccc}
\hline$M_{1}$ & $M_{2}$ & $M_{3}$ & $\mu$ & $\tan \beta$ & $m_{A^{0}}$ \\
\hline $139 \mathrm{GeV}$ & $264 \mathrm{GeV}$ & $800 \mathrm{GeV}$ & $120 \mathrm{GeV}$ & 10 & $800 \mathrm{GeV}$ \\
\hline \hline
\end{tabular}

(b) Neutralino and chargino masses.

\begin{tabular}{lccccc}
\hline$m_{\tilde{\chi}_{1}^{0}}$ & $m_{\tilde{\chi}_{2}^{0}}$ & $m_{\tilde{\chi}_{3}^{0}}$ & $m_{\tilde{\chi}_{4}^{0}}$ & $m_{\tilde{\chi}_{1}^{+}}$ & $m_{\tilde{\chi}_{2}^{+}}$ \\
\hline $87.0 \mathrm{GeV}$ & $133.1 \mathrm{GeV}$ & $158.3 \mathrm{GeV}$ & $310.1 \mathrm{GeV}$ & $109.3 \mathrm{GeV}$ & $310.1 \mathrm{GeV}$ \\
\hline \hline
\end{tabular}

(c) Important branching ratios.

\begin{tabular}{lcccc}
\hline$B\left(\tilde{g} \rightarrow c \bar{t} \tilde{\chi}_{1}^{0}\right)$ & $B\left(\tilde{g} \rightarrow c \bar{t} \tilde{\chi}_{2}^{0}\right)$ & $B\left(\tilde{g} \rightarrow b \bar{c} \tilde{\chi}_{1}^{+}\right)$ & $B\left(\tilde{\chi}_{2}^{0} \rightarrow \tilde{\chi}_{1}^{0} \nu \bar{\nu}\right)$ & $B\left(\tilde{\chi}_{1}^{+} \rightarrow \mu^{+} \nu_{\mu} \tilde{\chi}_{1}^{0}\right)$ \\
\hline $3.4 \%$ & $6.1 \%$ & $11.2 \%$ & $18.4 \%$ & $11.2 \%$ \\
\hline \hline
\end{tabular}


TABLE V. Weak scale parameters at $Q=1 \mathrm{TeV}$ (except for $m_{A^{0}}$ being the pole mass), the corresponding neutralino and chargino masses and some important branching ratios for a scenario with a winolike LSP, where $m_{\tilde{g}}=972 \mathrm{GeV}$.

\begin{tabular}{lccccc}
\hline \hline (a) Weak scale parameters at $Q=1 \mathrm{TeV}$. & & \\
\hline$M_{1}$ & $M_{2}$ & $M_{3}$ & $\mu$ & $\tan \beta$ & $m_{A^{0}}$ \\
\hline $400 \mathrm{GeV}$ & $300 \mathrm{GeV}$ & $800 \mathrm{GeV}$ & $350 \mathrm{GeV}$ & 10 & $800 \mathrm{GeV}$ \\
\hline \hline
\end{tabular}

(b) Neutralino and chargino masses.

\begin{tabular}{lccccc}
\hline$m_{\tilde{\chi}_{1}^{0}}$ & $m_{\tilde{\chi}_{2}^{0}}$ & $m_{\tilde{\chi}_{3}^{0}}$ & $m_{\tilde{\chi}_{4}^{0}}$ & $m_{\tilde{\chi}_{1}^{+}}$ & $m_{\tilde{\chi}_{2}^{+}}$ \\
\hline $275.5 \mathrm{GeV}$ & $362.6 \mathrm{GeV}$ & $376.4 \mathrm{GeV}$ & $433.1 \mathrm{GeV}$ & $280.0 \mathrm{GeV}$ & $407.1 \mathrm{GeV}$ \\
\hline \hline
\end{tabular}

(c) Important branching ratios.

\begin{tabular}{lcccc}
\hline$B\left(\tilde{g} \rightarrow c \bar{t} \tilde{\chi}_{1}^{0}\right)$ & $B\left(\tilde{g} \rightarrow c \bar{t} \tilde{\chi}_{2}^{0}\right)$ & $B\left(\tilde{g} \rightarrow b \bar{c} \tilde{\chi}_{1}^{+}\right)$ & $B\left(\tilde{\chi}_{2}^{0} \rightarrow \tilde{\chi}_{1}^{0} \nu \bar{\nu}\right)$ & $B\left(\tilde{\chi}_{1}^{+} \rightarrow \mu^{+} \nu_{\mu} \tilde{\chi}_{1}^{0}\right)$ \\
\hline $2.5 \%$ & $6.3 \%$ & $5.8 \%$ & $4.1 \%$ & $13.2 \%$ \\
\hline \hline
\end{tabular}

$\left.b \bar{c} e^{+} \nu_{e} \tilde{\chi}_{1}^{0}\right)=B\left(\tilde{g} \rightarrow \bar{b} c e^{-} \bar{\nu}_{e} \tilde{\chi}_{1}^{0}\right)=0.8 \%$. Hence, the signature $b \bar{c}$ (or $\bar{b} c$ ) plus a lepton $\left(\mu^{ \pm}\right.$or $\left.e^{ \pm}\right)$plus $E_{\mathrm{T}}^{\text {mis }}$ has a probability of about $3 \%$.

Summarizing the discussion of this section we can say that the branching ratios of the QFV three-particle gluino decays depend not only on the generation mixing in the squark sector, but also quite strongly on the parameters of the neutralino/chargino sector.

\section{MEASURABILITY OF THE QFV GLUINO THREE-BODY DECAYS}

We calculate the relevant gluino production cross sections at leading order using the WHIZARD/O'MEGA packages [37,38] where we have implemented the model described in Sec. II with squark generation mixing in its most general form. We use the CTEQ6L global parton density fit [39] for the parton distribution functions and take $Q=m_{\tilde{p}_{1}}+m_{\tilde{p}_{2}}$ for the factorization scale, where $\tilde{p}_{1}$ and $\tilde{p}_{2}$ are the sparticle pair produced. The QCD coupling $\alpha_{s}(Q)$ is also evaluated (at the two-loop level) at this scale $Q$. Because of the heavy squarks in our reference scenario of Table II, the dominant gluino production process at LHC is $p p \rightarrow \tilde{g} \tilde{g} X$, where $X$ contains beam-jets only. For the scenario of Table II the corresponding cross section is practically independent of $\delta_{23}^{u R R}$ and is about $170 \mathrm{fb}$ $(3 \mathrm{fb})$ at $\sqrt{s}=14 \mathrm{TeV}(7 \mathrm{TeV})$. (Note that $m_{\tilde{g}}=$ $975 \mathrm{GeV}$ and $972 \mathrm{GeV}$ for $\delta_{23}^{u R R}=0$ and 0.8 , respectively.) The sum of the cross sections of the other gluino production processes, such as $p p \rightarrow \tilde{g} \tilde{q}_{1} X, \tilde{g} \tilde{q}_{2} X, \tilde{g} \tilde{\chi}_{1}^{0} X, \tilde{g} \tilde{\chi}_{2}^{0} X$, is 2 orders of magnitude smaller than that of $p p \rightarrow \tilde{g} \tilde{g} X$.

In Fig. 9 we show the signal rates due to $p p \rightarrow \tilde{g} \tilde{g} X$, with $X$ containing beam-jets only, at $\sqrt{s}=14 \mathrm{TeV}$, where at least one of the pair-produced gluinos decays as $\tilde{g} \rightarrow c \bar{t}(\bar{c} t) \tilde{\chi}_{1}^{0}$, as a function of $\delta_{23}^{u R R}$ for the scenario of Table II. All the constraints mentioned in Sec. II are satisfied in the range $\left|\delta_{23}^{u R R}\right| \lesssim 0.85$. The rate of the final state $\operatorname{cc} \bar{t} \bar{t} E_{\mathrm{T}}^{\text {mis }} X$, produced in the case when both gluinos decay like $\tilde{g} \rightarrow c \bar{t} \tilde{\chi}_{1}^{0}$, reaches $7 \mathrm{fb}$ for $\left|\delta_{23}^{u R R}\right|=0.8$ (full blue line), yielding 700 events for an integrated luminosity of $100 \mathrm{fb}^{-1}$. The charge conjugated final state $\bar{c} \bar{c} t t E_{\mathrm{T}}^{\mathrm{mis}} X$ has the same rate. The full red line shows the rate for the QFV case where one gluino decays as $\tilde{g} \rightarrow c \bar{t} \tilde{\chi}_{1}^{0}$ and the other one as $\tilde{g} \rightarrow \bar{c} t \tilde{\chi}_{1}^{0}$ plus the QFC case with one $\tilde{g} \rightarrow c \bar{c} \tilde{\chi}_{1}^{0}$ and the other $\tilde{g} \rightarrow t \bar{t} \tilde{\chi}_{1}^{0}$. The dashed red line presents the rate for the QFC case only. One can see that the QFV signal has a rate of about $14 \mathrm{fb}$ for $\left|\delta_{23}^{u R R}\right|=$ 0.8 . The green lines show the case of one gluino decaying as $\tilde{g} \rightarrow c \bar{t} \tilde{\chi}_{1}^{0}$, and the other one as $\tilde{g} \rightarrow c \bar{c} \tilde{\chi}_{1}^{0}$ (full green line) or $\tilde{g} \rightarrow t \bar{t} \tilde{\chi}_{1}^{0}$ (dashed green line). These rates reach $25 \mathrm{fb}$ and $10 \mathrm{fb}$, respectively, for $\left|\delta_{23}^{u R R}\right|=0.8$. The charge conjugated final states have the same rates.

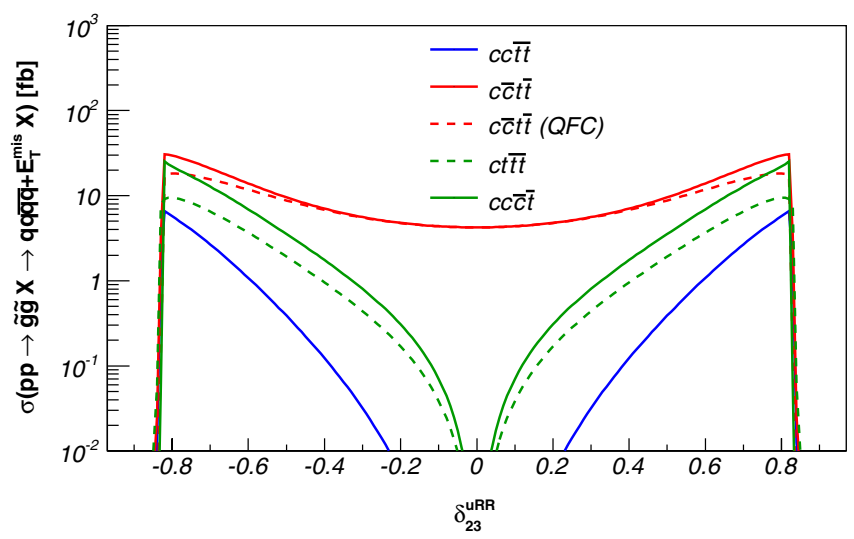

FIG. 9 (color online). Signal rates for $p p \rightarrow \tilde{g} \tilde{g} X$ at $\sqrt{s}=$ $14 \mathrm{TeV}$ where at least one of the gluinos decays as $\tilde{g} \rightarrow$ $c \bar{t}(\bar{c} t) \tilde{\chi}_{1}^{0}$, as a function of $\delta_{23}^{u R R}$ with the other QFV parameters being zero and the other parameters fixed as in Table II. Shown are the rates for the final states with $c c \bar{t} \bar{t} E_{\mathrm{T}}^{\mathrm{mis}}$ (lower solid line), $c \bar{c} t \bar{t} E_{\mathrm{T}}^{\mathrm{mis}}(\mathrm{QFV}+\mathrm{QFC})$ (upper solid line), $c \bar{c} t \bar{t} E_{\mathrm{T}}^{\mathrm{mis}}$ (QFC only) (upper dashed line), $c t \bar{t} \bar{t} E_{\mathrm{T}}^{\mathrm{mis}}$ (lower dashed line), $c c \bar{c} \bar{t} E_{\mathrm{T}}^{\mathrm{mis}}$ (middle solid line). 


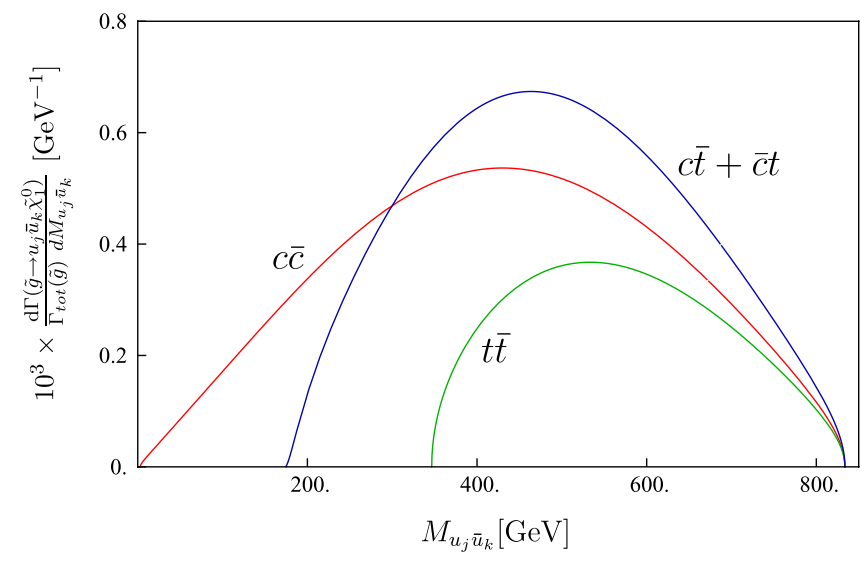

FIG. 10 (color online). Invariant mass distributions of two uptype quarks from the decay $\tilde{g} \rightarrow u_{j} \bar{u}_{k} \tilde{\chi}_{1}^{0}$, with $\delta_{23}^{u R R}=0.8$, the other QFV parameters being zero, and the other parameters fixed as in Table II.

A characteristic feature of a three-particle decay of the gluino is the invariant mass distribution of the two produced quarks. We calculate the invariant mass distributions $\mathrm{d} \Gamma\left(\tilde{g} \rightarrow u_{j} \bar{u}_{k} \tilde{\chi}_{1}^{0}\right) /\left(\Gamma_{\text {tot }}(\tilde{g}) d M_{u_{j} \bar{u}_{k}}\right)$, where $M_{u_{j} \bar{u}_{k}}$ is the invariant mass of the two-quark system $u_{j} \bar{u}_{k}, M_{u_{j} \bar{u}_{k}}^{2}=$ $\left(p_{u_{j}}+p_{\bar{u}_{k}}\right)^{2}$. In Fig. 10 we show these distributions for $\tilde{g} \rightarrow t \bar{t} \tilde{\chi}_{1}^{0}, c \bar{c} \tilde{\chi}_{1}^{0}, c \bar{t} \tilde{\chi}_{1}^{0}+\bar{c} t \tilde{\chi}_{1}^{0}$ with $\delta_{23}^{u R R}=0.8$ and the other QFV parameters being zero for the scenario of Table II. In contrast to the case where the squarks are lighter than the gluino and the gluino decays via real squarks [11], no edge structure appears. However, the thresholds and the shapes of the distributions are very different. The endpoint is at $\left(m_{\tilde{g}}-m_{\tilde{\chi}_{1}^{0}}\right)$. The thresholds are at $2 m_{c}, m_{c}+m_{t}$ and $2 m_{t}$, respectively. Measuring these distributions could be helpful for separating the $\mathrm{QFV}$ decays into $c \bar{t} \tilde{\chi}_{1}^{0}+\bar{c} t \tilde{\chi}_{1}^{0}$ from the $\mathrm{QFC}$ decays.

A typical $\tilde{g} \tilde{g}$ production event has at least four large- $p_{T}$ jets and large $E_{\mathrm{T}}^{\mathrm{mis}}$. An event with a QFV gluino decay $\tilde{g} \rightarrow c \bar{t} \tilde{\chi}_{1}^{0}\left(\bar{c} t \tilde{\chi}_{1}^{0}\right)$ should contain at least one top (antitop) quark in the final state, which must be identified. This is possible by using the decay $t \rightarrow b W^{ \pm}$with the $W^{ \pm}$decaying into two jets. For this purpose a special method was proposed in [40]. Charm tagging would be extremely helpful for a clear identification of the QFV gluino decay $\tilde{g} \rightarrow \bar{c} t \tilde{\chi}_{1}^{0}\left(c \bar{t} \tilde{\chi}_{1}^{0}\right)$. If this is not possible one could search for the decay $\tilde{g} \rightarrow \bar{q} t \tilde{\chi}_{1}^{0}\left(q \bar{t} \tilde{\chi}_{1}^{0}\right), q \neq t$. Typical signatures of QFV $\tilde{g}$ pair events are: $t($ or $\vec{t})+3$ jets $+E_{\mathrm{T}}^{\text {mis }}+X, t+$ $t($ or $\bar{t}+\bar{t})+2$ jets $+E_{\mathrm{T}}^{\mathrm{mis}}+X$ and $t+t+\bar{t}($ or $\bar{t}+\bar{t}+t)+$ 1 jet $+E_{\mathrm{T}}^{\mathrm{mis}}+X$, where $X$ contains beam-jets only. Note, that the signal events $t+t($ or $\bar{t}+\bar{t})+2$ jets $+E_{\mathrm{T}}^{\mathrm{mis}}+X$ can practically not be produced in the MSSM (nor in the SM) with QFC.

The rate of a possible SUSY background from pair production of squarks, such as $p p \rightarrow \tilde{q} \overline{\tilde{q}} X$ with $\tilde{q} \rightarrow c \tilde{\chi}_{1}^{0}, \overline{\tilde{q}} \rightarrow \bar{t} \tilde{\chi}_{1}^{0}$ is much smaller than that of the signal of gluino pair production due to the larger squark masses. As shown in the SUSY searches by ATLAS and CMS $[12,13]$, the SM backgrounds, such as QCD multijets, $W^{ \pm}+$jets, $Z^{0}+$ jets, $t \bar{t}$ and single top production, can be strongly reduced by appropriate selection cuts.

\section{CONCLUSIONS}

We have studied QFV decays of gluino within the MSSM in the case that all squarks are heavier than the gluino and, hence, the gluino has only three-particle decays. Starting from the most general squark mass matrix, we have assumed mixing between the second and the third squark generations in the up and down sectors. We have taken into account all relevant experimental constraints from SUSY particles and Higgs searches as well as from precision data in the $\mathrm{B}$ meson sector. Furthermore we have respected the vacuum stability conditions for the trilinear coupling matrices. It has turned out that of all QFV parameters the parameters $\delta_{23}^{u R R}, \delta_{23}^{d R R}$ and $\delta_{23}^{L L}$ play the most important role in our study. We have concentrated on the QFV decays $\tilde{g} \rightarrow c \bar{t}(\bar{c} t) \tilde{\chi}_{1}^{0}$ and $\tilde{g} \rightarrow b \bar{s}(\bar{b} s) \tilde{\chi}_{1}^{0}$ which presumably have the clearest signatures for the presence of QFV in the MSSM. We have studied these within a prototype scenario with gluino mass $m_{\tilde{g}} \approx 1 \mathrm{TeV}$ and squarks with masses between $\sim 1 \mathrm{TeV}$ and $\sim 3 \mathrm{TeV}$, where the lightest squarks are $\tilde{c}_{R}-\tilde{t}_{R}$ mixtures (or $\tilde{s}_{R}-\tilde{b}_{R}$ mixtures). These QFV decay branching ratios can reach up to $40 \%$ (35\%). We have paid special attention to the dependence of the QFV decay branching ratios on the chargino/neutralino parameters. In this context we have considered three cases where the lightest neutralino is bino-, wino- and Higgsino-like, respectively. We have found that the QFV decay branching ratios depend strongly also on the chargino/neutralino parameters.

As in our scenario the squarks are heavier than the gluino, the dominant gluino production process at LHC is gluino pair production, $p p \rightarrow \tilde{g} \tilde{g} X$. We have calculated the rates for the various signatures stemming from QFV gluino decays as well as the invariant mass distributions of the two-quark system $c \bar{t}+\bar{c} t, c \bar{c}, t \bar{t}$ in the final states. We have found that the rates of the resulting QFV signatures, like $p p \rightarrow t c \bar{c} \bar{c} E_{\mathrm{T}}^{\mathrm{mis}} X$ and $p p \rightarrow t t \bar{c} \bar{c} E_{\mathrm{T}}^{\text {mis }} X$, are significant at LHC. This could have an important influence on the search for gluinos and the determination of the basic MSSM parameters at LHC.

\section{ACKNOWLEDGMENTS}

This work is supported by the "Fonds zur Förderung der wissenschaftlichen Forschung (FWF)" of Austria, Project No. I 297-N16, and by the DFG, Project No. PO-1337/2-1. B.H. acknowledges support by the LandesExzellenzinitiative Hamburg. 
[1] C. Amsler et al. (Particle Data Group), Phys. Lett. B 667, 1 (2008).

[2] T. Plehn, M. Rauch, and M. Spannowsky, Phys. Rev. D 80, 114027 (2009).

[3] A. J. Buras, P. Gambino, M. Gorbahn, S. Jager, and L. Silvestrini, Phys. Lett. B 500, 161 (2001).

[4] G. D'Ambrosio, G. F. Giudice, G. Isidori, and A. Strumia, Nucl. Phys. B645, 155 (2002).

[5] A. L. Kagan, G. Perez, T. Volansky, and J. Zupan, Phys. Rev. D 80, 076002 (2009).

[6] G. Bozzi, B. Fuks, B. Herrmann, and M. Klasen, Nucl. Phys. B787, 1 (2007).

[7] B. Fuks, B. Herrmann, and M. Klasen, Nucl. Phys. B810, 266 (2009).

[8] A. Bartl, H. Eberl, B. Herrmann, K. Hidaka, W. Majerotto, and W. Porod, Phys. Lett. B 698, 380 (2011).

[9] M. Bruhnke, B. Herrmann, and W. Porod, J. High Energy Phys. 09 (2010) 006.

[10] T. Hurth and W. Porod, J. High Energy Phys. 08 (2009) 087.

[11] A. Bartl, K. Hidaka, K. Hohenwarter-Sodek, T. Kernreiter, W. Majerotto, and W. Porod, Phys. Lett. B 679, 260 (2009).

[12] G. Aad et al. (ATLAS Collaboration), Phys. Rev. Lett. 106, 131802 (2011); G. Aad et al. (ATLAS Collaboration), Phys. Lett. B 701, 186 (2011); 701, 398 (2011); Eur. Phys. J. C 71, 1 (2011); S. Caron (ATLAS Collaboration), in 46th Rencontres de Moriond on Electroweak Interactions and Unified Theories, La Thuile, Italy, 2011 (unpublished).

[13] V. Khachatryan et al. (CMS Collaboration), Phys. Lett. B 698, 196 (2011); S. Chatrchyan et al. (CMS Collaboration),J. High Energy Phys. 06 (2011) 026; S. Chatrchyan et al. (CMS Collaboration), arXiv:1106.4503 [J. High Energy Phys. (to be published)]; S. Chatrchyan et al. (CMS Collaboration), arXiv:1107.1279 [Phys. Rev. $\mathrm{D}$ (to be published)]; S. Chatrchyan et al. (CMS Collaboration), J. High Energy Phys. 07 (2011) 113.

[14] W. Ehrenfeld, in 19th International Conference on Supersymmetry and Unification of Fundamental Interactions (SUSY2011), Fermilab, Batavia, 2011 (unpublished).

[15] I. Melzer-Pellmann, in 19th International Conference on Supersymmetry and Unification of Fundamental Interactions (SUSY2011)" Fermilab, Batavia, 2011 (unpublished); S. Chatrchyan et al. (CMS Collaboration), arXiv:1109.2352 [Phys. Rev. Lett. (to be published)].

[16] M. Flowerdew, 19th International Conference on Supersymmetry and Unification of Fundamental Interactions (SUSY2011), Fermilab, Batavia, 2011 (unpublished).

[17] B. C. Allanach et al., Comput. Phys. Commun. 180, 8 (2009).
[18] F. Gabbiani, E. Gabrielli, A. Masiero, and L. Silvestrini, Nucl. Phys. B477, 321 (1996).

[19] W. S. Hou, Phys. Rev. D 48, 2342 (1993).

[20] M. Carena, A. Menon, R. Noriega-Papaqui, A. Szynkman, and C. E. M. Wagner, Phys. Rev. D 74, 015009 (2006); see also P. Ball and R. Fleischer, Eur. Phys. J. C 48, 413 (2006).

[21] K. Trabelsi, Proc. Sci., ICHEP2010 (2010) 566.

[22] M. Misiak et al., Phys. Rev. Lett. 98, 022002 (2007); see also T. Hurth, E. Lunghi, and W. Porod, Nucl. Phys. B704, 56 (2005).

[23] M. Iwasaki et al. (Belle Collaboration), Phys. Rev. D 72, 092005 (2005); B. Aubert et al. (BABAR Collaboration), Phys. Rev. Lett. 93, 081802 (2004).

[24] T. Huber, T. Hurth, and E. Lunghi, Nucl. Phys. B802, 40 (2008).

[25] G. Borissov, Proc. Sci., ICHEP2010 (2010) 531.

[26] S. Schael et al. (ALEPH Collaboration, DELPHI Collaboration, L3 Collaboration, OPAL Collaboration, and LEP Working Group for Higgs Boson Searches), Eur. Phys. J. C 47, 547 (2006).

[27] B. C. Allanach, A. Djouadi, J. L. Kneur, W. Porod, and P. Slavich, J. High Energy Phys. 09 (2004) 044.

[28] J. A. Casas and S. Dimopoulos, Phys. Lett. B 387, 107 (1996).

[29] E. Shabalina, Proc. Sci., ICHEP2010 (2010) 561.

[30] M. L. Vazquez Acosta, in 19th International Conference on Supersymmetry and Unification of Fundamental Interactions (SUSY2011), Fermilab, Batavia, 2011 (unpublished); S. Chatrchyan et al. (CMS Collaboration), Phys. Rev. Lett. 106, 231801 (2011); G. Aad et al. (ATLAS Collaboration), arXiv:1107.5003 [Phys. Lett. B (to be published)].

[31] G. Altarelli, R. Barbieri, and F. Caravaglios, Int. J. Mod. Phys. A 13, 1031 (1998).

[32] G. Sguazzoni, Nucl. Phys. B, Proc. Suppl. 117, 709 (2003); P. Lutz, Nucl. Phys. B, Proc. Suppl. 117, 735 (2003).

[33] W. Porod, Comput. Phys. Commun. 153, 275 (2003); The code SPheno v3.0 can be obtained from: http://www .physik.uni-wuerzburg.de/ porod/SPheno.html.

[34] W. Porod and F. Staub, arXiv:1104.1573.

[35] J. A. Aguilar-Saavedra et al., Eur. Phys. J. C 46, 43 (2006).

[36] S. Heinemeyer, W. Hollik, F. Merz, and S. Penaranda, Eur. Phys. J. C 37, 481 (2004).

[37] W. Kilian, T. Ohl, and J. Reuter, Eur. Phys. J. C 71, 1742 (2011).

[38] M. Moretti, T. Ohl, and J. Reuter, arXiv:hep-ph/0102195.

[39] J. Pumplin et al., J. High Energy Phys. 07 (2002) 012.

[40] J. Hisano, K. Kawagoe, R. Kitano, and M. M. Nojiri, Phys. Rev. D 66, 115004 (2002); J. Hisano, K. Kawagoe, and M. M. Nojiri, Phys. Rev. D 68, 035007 (2003). 